\title{
An Improved Method for the Segmentation of Roots from X-ray Computed Tomography 3D Images: Rootine v.2
}

Maxime Phalempin ( $\square$ maxime.phalempin@ufz.de)

UmweltForschungZentrum https://orcid.org/0000-0003-1198-807X

Eva Lippold

UFZ Umweltforschungszentrum Leipzig-Halle: Helmholtz-Zentrum fur Umweltforschung UFZ

Doris Vetterlein

UFZ Umweltforschungszentrum Leipzig-Halle: Helmholtz-Zentrum fur Umweltforschung UFZ

\section{Steffen Schlüter}

UFZ Umweltforschungszentrum Leipzig-Halle: Helmholtz-Zentrum fur Umweltforschung UFZ

\section{Methodology}

Keywords: High-throughput root phenotyping, Image analysis, Root segmentation, Root system architecture, Cylindrical feature detection, X-ray computed tomography , Root diameter

Posted Date: October 27th, 2020

DOl: https://doi.org/10.21203/rs.3.rs-96550/v1

License: (c) (i) This work is licensed under a Creative Commons Attribution 4.0 International License. Read Full License

Version of Record: A version of this preprint was published at Plant Methods on April 8th, 2021. See the published version at https://doi.org/10.1186/s13007-021-00735-4. 


\section{An improved method for the segmentation of roots from X-ray computed tomography 3D images: Rootine v.2}

${ }^{1}$ Helmholtz Centre for Environmental Research GmbH-UFZ; Department of Soil System Science,

3 Halle (Saale), Germany.

$4 \quad{ }^{2}$ Martin-Luther-University Halle-Wittenberg; Institute of Agricultural and Nutritional Sciences, Halle (Saale), Germany.

\section{Corresponding author}

6 Maxime Phalempin (maxime.phalempin@ufz.de)

\section{Abstract}

\section{Background}

9 X-ray computed tomography is acknowledged as a powerful tool for the study of root

10 system architecture of plants growing in soil. In this paper, we improved the original root

11 segmentation algorithm "Rootine" and present its succeeding version "Rootine v.2". In

12 addition to grey value information, Rootine algorithms are based on shape detection of

13 cylindrical roots. Both algorithms are macros for the ImageJ software and are made

14 freely available to the public. New features in Rootine v.2 are (1) a pot wall detection

15 and removal step to avoid segmentation artefacts for roots growing along the pot wall,

16 (2) a calculation of the root average grey value based on a histogram analysis, (3) an

17 automatic calculation of thresholds for hysteresis thresholding of the tubeness image to

18 reduce the number of parameters and (4) a false negatives recovery based on shape 
19 criteria to increase root recovery. We compare the segmentation results of Rootine v. 1

20 and Rootine v.2 with the results of root washing and subsequent analysis with

21 WinRhizo. We use a benchmark dataset of maize roots (Zea mays L. cv. B73) grown in

22 repacked soil for two scenarios with differing soil heterogeneity and image quality.

\section{Results}

24 We demonstrate that Rootine v.2 outperforms its preceding version in terms of root

25 recovery and enables to match better the root diameter distribution data obtained with

26 root washing. Despite a longer processing time, Rootine v.2 comprises less user-

27 defined parameters and shows an overall greater usability.

\section{Conclusion}

29 The proposed method facilitates higher root detection accuracy than its predecessor

30 and has the potential for improving high-throughput root phenotyping procedures based

31 on X-ray CT data analysis.

\section{Keywords}

33 High-throughput root phenotyping - Image analysis - Root segmentation - Root system architecture -

34 Cylindrical feature detection - X-ray computed tomography - Root diameter

\section{Abbreviations}

36 Computed Tomography (CT); Root System Architecture (RSA); Magnetic Resonance

37 Imaging (MRI); Non-Local Means (NLM); Region of Interest (ROI); Root Length Density

38 (RLD); Absolute Difference Transform (ADT); WinRHIZO (WR); 


\section{Introduction}

40 X-ray computed tomography (CT) is acknowledged as a powerful tool for the

41 study of root system architecture (RSA) of plants growing in soil. However, the study of

42 the root system architecture is only possible after performing root segmentation, i.e. the

43 binarisation of the greyscale data into root voxels and background voxels. Root

44 segmentation is often regarded as a tedious and difficult task as its success depends on

45 several factors such as the image resolution, the signal to noise ratio during image

46 acquisition and the grey value contrast between the roots and all other surrounding

47 features in soil [1].

48 In the past years, many methods have been developed to segment and visualise

49 roots in tomograms acquired with X-ray CT [1-16]. Some algorithms rely on simple

50 thresholding methods [8]. With these methods, the roots are segmented based on a

51 histogram analysis and a grey value criterion. These methods usually fail at segmenting

52 roots properly because of the overlapping grey value of roots, water, organic matter and

53 the soil matrix. The grey value at the edges of roots, water, organic matter and soil

54 matrix also show gradual changes of intensity spanning several voxels rather than a

55 crisp intensity step [17]. This effect, known as the "partial volume effect", is also

56 responsible for poor segmentation results when using simple thresholding methods.

57 More advanced thresholding methods rely on the use of adaptive local thresholding

58 values (also referred to as "Region growing") which use an additional connectivity

59 criterion to binarise the root and background voxels [3]. For both the simple and the

60 adaptive thresholding methods, there is an inherent trade-off to be made by the user. If 
61 the grey value range assigned to roots bounded by two thresholds is too broad,

62 oversegmentation may occur (i.e. segmented root edges extend into the surrounding

63 features and appear frayed) and the false positives need to be removed through user-

64 interaction which is a subjective, tedious and time-consuming task. Inversely, if this grey

65 value range is too narrow, an important loss of roots may occur which biases the RSA

66 analysis of the scanned sample. To tackle the issue of overlapping grey values of roots

67 and other materials, root tracking methods such as the "RooTrak" algorithm have been

68 developed [10]. With this method, the volumetric data is viewed as a sequence of $x-y$

69 cross-sectional images aligned along the $z$ axis. As the 3D stack is explored, root cross

70 sections appear to move in the image and such "movements" can be used to

71 reconstruct the root system. Methods relying on deep-learning algorithms and multi-

72 scaled based approaches have also become common in X-ray CT and MRI imaging.

73 Promising applications of deep-learning for the segmentation of roots from X-ray CT

74 data were recently demonstrated by [13].

75 Gao et al. [6] proposed a new algorithm to segment root systems growing in soil

76 by exploiting a typical morphological characteristic of the roots, i.e. their cylindrical

77 shape. This approach was first introduced for vessel detection in medical imaging [18].

78 The vessel enhancement filter was later adopted to segment roots in 3D Magnetic

79 Resonance Imaging (MRI) data [19]. The rationale of this method is that the cylindrical

80 shape of roots is unique among all materials and features found in soil. The shape-

81 based semi-automated algorithm is named "Rootine" [6] and has shown to outperform

82 the "Root1" [4] and "Region growing" [3] methods in terms of root recovery and 
83 segmentation accuracy. This demonstrated promising future applications of the

84 algorithm for high-throughput root phenotyping based on X-ray CT data analysis.

85 However, the Rootine algorithm relies on a substantial number of parameters to be

86 calibrated by the user. Moreover, Rootine suffers from the fact that some of the

87 parameters and their effects are difficult to identify and to interpret by a non-

88 experienced user.

89 In this paper, we aim at developing an improved method for the segmentation of

90 roots from 3D X-ray CT images that overcomes the aforementioned drawbacks of

91 Rootine. The objectives of this work are then to develop a new Rootine version, i.e.

92 "Rootine v.2", segmentation algorithm for which the segmentation accuracy and the

93 user friendliness are increased. Specific objectives are to propose a second version in

94 which:

95 (1) the root recovery is higher;

96 (2) the segmented root diameters are better captured;

97 (3) segmentation artefacts are reduced;

98 (4) the number of tunable parameters is reduced;

99 (5) the parameters are related to root properties (i.e. their grey value, shape and 100 connectivity).

101 The ability of the new segmentation algorithm to fulfill these criteria is evaluated by 102 systematic comparison with the former algorithm Rootine (which will be referred to as

103 "Rootine v.1") and the results obtained by conventional, destructive root sampling and 104 analysis of the washed-off roots with the software WinRHIZO. The comparison with 
105 other state-of-the-art method is not carried out again, as the previous version of Rootine

106 already demonstrated to outperform them [6]. In addition, a comparison between both

107 algorithms is made by considering aspects such as the runtime and the overall usability

108 of the algorithms. In that respect, the benchmark dataset of the "worse case" scenario

109 presented in [6] and briefly described in the method section of this paper is used. This

110 benchmark dataset was chosen to test Rootine v.2 as it presents several challenges to

111 overcome, namely the presence of high soil heterogeneity, a poor quality of the images

112 (i.e. a low number of projections during image acquisition) and a rather low image

113 resolution as compared to the diameter of the roots to segment. The two first challenges

114 contributed to a deterioration of the signal to noise ratio whereas the third exacerbates

115 the partial volume effect at the edges of the fine roots. In the study of [6], these

116 challenges led to a rather low root recovery of (i.e. $29 \%$ ) in comparison with

117 conventional root sampling. These challenges combined make the benchmark dataset

118 of [6] a perfect candidate for further testing and improvements of root segmentation

119 algorithms. In order to show that the improvements in segmentation quality are not

120 solely due to an overfitting of the Rootine v.2 for this particular dataset, we also

121 demonstrate the performance of the new version on the so called "best case" scenario

122 dataset of [6]. In this "best case" scenario, the soil and the scan settings were chosen in

123 order to create low soil heterogeneity and a high signal to noise ratio. Those two

124 aspects contributed to a robust estimation of root length (i.e. $99 \%$ of recovery) in

125 comparison with root washing and analysis with WinRHIZO. 


\section{Material and Methods}

\section{Plant growth conditions and destructive sampling}

128 Maize plants (Zea mays L. cv. B73) were grown in repacked soil sieved down to

$129 \leq 2 \mathrm{~mm}$ particle size. The plants were grown in a climate chamber for 21 days in

130 cylindrical containers of $7 \mathrm{~cm}$ inner diameter and $23 \mathrm{~cm}$ height. Six plants were

131 analyzed for each scenario. One day after X-ray CT scanning, the plants were

132 harvested and the pots were cut in several layers of $4 \mathrm{~cm}$. The roots in those layers

133 were washed off with deionised water and stored in a $50 \%$ ethanol solution prior to

134 analysis. In order to assess root length density (RLD) for each layer, root samples were

135 scanned with a flatbed scanner (EPSON perfection V700) and the obtained images

136 were analyzed with WinRHIZO Pro ${ }^{\mathrm{TM}}$ (Version 2019a, Regent Instruments, Canada). In

137 total, twelve layers were investigated for each scenario (i.e. 2 per growing pot, one at

138 the top and one at the bottom). For detailed information on the plant growth conditions

139 and the destructive root sampling method, the reader is referred to [6].

\section{Workflow of Rootine v.2}

141 The workflow of Rootine v.2 is synoptically shown in Fig. 1 where the novelties of

142 the algorithm are highlighted in blue and the steps and/or parameters that were

143 modified from the original Rootine v.1 are shown in purple.

144 Image acquisition

145 3D X-ray CT images were acquired and reconstructed into an 8-bit greyscale 3D 146 tomogram having a voxel size of $45 \mu \mathrm{m}$. The 8-bit conversion allows saving space 
147 without considerable loss of information. During the 8-bit conversion, contrast was

148 optimized using a percentile stretching method, i.e. $0.2 \%$ of the darkest and brightest

149 voxels are set to 0 and 255 respectively. A linear stretching is applied for all grey values

150 between 0 and 255 . Considering the geometry of the panel detector of the X-ray CT

151 device (X-TEK XTH 225, Nikon Metrology), pots were scanned at two depth intervals

152 (i.e. a bottom and top depth) making sure that an overlapping region was present. For

153 more information regarding the image acquisition procedure, the reader is here again

154 referred to [6].

155 Preprocessing

156 Before concatenation of the bottom and the top images, the overlapping regions

157 in both scans are removed using the "Slice remover" function available in the free

158 software ImageJ [20]. After concatenation, the obtained image comprises 3000 voxels

159 in the $z$ dimension, 1750 voxels in the $x$ and $y$ dimension and has a size $\approx 8.6$ GB. The

160 vertical extent of the stack is $13.5 \mathrm{~cm}$. At the boundary of the two stitched images in the

161 concatenated stack, a grey value discontinuity is present due to an illumination drift

162 caused by the CT hardware (see Fig. S2 in [6]). This grey value discontinuity is

163 corrected for using the "Attenuation correction" plugin [21] in ImageJ. This correction

164 applies a linear transformation of grey values to each slice of the stack in order to make 165 the average and standard deviation of the background constant (i.e. equal to that of a 166 reference slice) throughout the stack [21]. Note that this grey value discontinuity is 167 specific to the CT hardware used in the benchmark dataset of [6] and that this step 168 might not be necessary with other set-ups. 
Once the grey value discontinuity of the stitched stack is corrected for, a filtering

170 step is performed with a "3D Non-local Means (NLM)" filter [22]. This filtering step is

171 performed with a plugin available in the ITK library [23]. This filter was chosen as it is

172 fast [22] and can easily be incorporated in the workflow thanks to its standalone

173 application. Note that in contrast to [6] (see Fig. S3 in [6]), we converted the images to

174 16-bit and added a constant grey value offset of 50000 to avoid the change of contrast

175 inherent to the use of this filter. The change in contrast is an outcome of the Rician

176 noise model implemented in this filter [22] as it was originally implemented for MRI

177 images. Avoiding the non-linear contrast enhancement for the low attenuation materials

178 makes the results directly comparable to other softwares implementing a 3D NLM filter

179 (e.g. Avizo $^{\mathrm{TM}}$ ). The strength of the filtering is determined by the parameter "Contrast

180 threshold" $\left(\mathrm{t}_{\text {con }}\right)$ which needs to be given by the user as an input parameter. It is

181 adjusted to the standard deviation of the image noise assessed by the histogram

182 analysis. Similarly as in [6], the remaining parameters of the 3D NLM filter were set to

183 default. The result of the 3D NLM filtering can be assessed by comparing the original

184 greyscale data (Fig. 2a) and the filtered image (Fig. 2b).

185 Rootine v.2 features a new pot wall detection step. This step serves two 186 purposes. The first purpose is to create a mask which will exclude the pot wall from the 187 data to segment and the second is to use the characteristic grey value of the pot wall to 188 generate a peak in the histogram. The generated peak is used later on for the 189 calculation of the average root grey value $\left(\bar{v}_{r}\right)$ during a background removal step. In 190 order to create a mask which excludes the pot wall, the coordinates of a circular region 
191 of interest (ROI) bounded within the pot wall limit need to be defined manually at three $z$ 192 slices of the stack, i.e. at the first, the middle and the last slices. Those three sets of

193 coordinates need to be given as input. The coordinates of the bounded $\mathrm{ROI}$ for all $\mathrm{z}$ 194 slices are then linearly interpolated from the given $x-y$ coordinates of the bounded ROI 195 at the three $z$ slices. This allows creating a 3D mask, i.e. a mask whose boundaries in 196 the $x$ and $y$ dimensions will move as the stack is explored in the $z$ dimension. Creating a 197 3D mask is necessary to cope with pots being tilted during the X-ray CT scanning. Note 198 that, at the resolution used in the benchmark dataset and considering the pot height, a 199 certain tilt of the pots is always present. For tilted pots, a 2D mask would result either in 200 masking the roots growing along the pot wall or in the inclusion of the pot wall in the 201 data to segment. With the 3D mask, a logical "And" operation is used on the filtered 202 image in order to remove the pot wall from the data to segment. Once the bounded ROI 203 is calculated for all $z$ slices, an extended ROI is created by simple extension of the 204 bounded ROI by 50 voxels (Fig. 3.a). The extended ROI serves the purpose of including 205 the pot wall in the histogram analysis of the stack so that a characteristic peak is 206 generated. After extracting the histogram of the extended ROI, a function searches for 207 the maxima in the lowest part (i.e. from 0 to 128) and in the highest part (i.e. from 128 to 208 255) of the histogram. This function then retrieves the grey value of those maxima, i.e. 209 P1 and P2 which correspond to the average grey value of the pot wall and of the soil 210 matrix respectively (Fig. 3.b). Those two values are used further down in the workflow 211 during the background removal step. 
An edge enhancement step is then applied with the "Unsharp Mask" filter in

213 ImageJ. "Unsharp Mask" filters enhance the local contrast between root edges, the

214 surrounding soil matrix and pores [24]. The degree of edge enhancement is controlled

215 by two input parameters. "Blur radius" is the standard deviation of the blur radius of the

216 Gaussian filter kernel and "Mask weight" determines the strength of the filtering. The

217 result of the edge enhancement step can be assessed by comparing the image filtered

218 with 3D NLM (Fig. 2b) and the image after "Unsharp Mask" (Fig. 2c).

219 A new feature of the Rootine v.2 algorithm is to apply a background removal 220 step. During the background removal, every voxel whose grey value deviates too much 221 from the average root grey value is masked out. This operation is performed in a three 222 steps procedure. During the first step, a calculation of the root average grey value is 223 carried out using the previously identified characteristic peaks of the pot wall and of the 224 soil matrix. Assuming that a shift of P1 and P2 would result in similar shift of $\bar{v}_{r}, \bar{v}_{r}$ can 225 be calculated for every image of the dataset using Eq. 1,

$$
\bar{v}_{r}=\mathrm{f}_{\mathrm{r}} \cdot(\mathrm{P} 2-\mathrm{P} 1)+\mathrm{P} 1
$$

227 where $f_{r}$ is the root grey value factor which has to be determined a priori on a 228 representative test image. The first step allows coping with differently contrasted images 229 in the dataset. Differences in image contrast are due to the percentile stretching method 230 used during the 8-bit conversion when reconstructing the tomograms. Once $\bar{v}_{r}$ is 231 calculated, a second step named "Absolute Difference Transform" (ADT) is applied. The 232 rationale of this step is to brighten the grey value of the roots and darken all background 
233 voxels including pores and soil matrix, as both materials have a grey value different

234 than $\bar{v}_{r}$. For every voxel, this is done by computing Eq. 2,

$$
\mathrm{v}_{\mathrm{ADT}}=255-\left|\mathrm{v}_{\mathrm{f}}-\bar{v}_{r}\right|
$$

236 where $v_{A D T}$ is the voxel grey value after the ADT and $v_{f}$ is the voxel grey value prior 237 ADT. The background is then masked out by thresholding the image with a threshold 238 value $\left(\mathrm{t}_{A D T}\right)$ calculated with Eq. 3 ,

$$
t_{A D T}=255-\frac{\mathrm{R}_{\mathrm{r}}}{2}
$$

240 where $R_{r}$ is the root grey value range centered around $\bar{v}_{r}$. $R_{r}$ has to be determined a 241 priori on a representative test image and has to be given as a input parameter. The 242 image obtained after background removal serves as the input image for the subsequent 243 root segmentation step. This three-step procedure replaces the simple pore masking 244 step in Rootine v.1. The result of the background removal step can be assessed by 245 comparing the image after edge enhancement (Fig. 2c) and the image after background 246 removal (Fig. 2d).

\section{Root segmentation}

248 A specificity of the Rootine algorithms is to segment roots by exploiting one of 249 their inherent characteristics, i.e. their cylindrical shape. To do so, the "Tubeness filter" 250 available in ImageJ is used. In brief, the tubeness filter performs a smoothing of the 251 image and produces an image in which the grey values are directly related to how 252 similar an object is to a cylinder. Generally, a scaled approach is adopted, i.e. the same 253 image is filtered with Gaussian filters of different strength determined by their $\sigma$ values, 
254 segmented and then combined. For low $\sigma$ values, roots of small diameters will evoke 255 high grey values after tubeness filtering whereas bigger roots will either appear hollow 256 or display low grey values after tubeness filtering. Increasing the $\sigma$ values of the 257 tubeness filter then results in the opposite effect, i.e. roots of greater diameter appear 258 brighter whereas roots of smaller diameter will vanish. The obtained series of images 259 are then thresholded using the "3D Hysteresis thresholding" method available in the 3D 260 ImageJ Suite [25] and then combined to reconstitute the full root system. Hysteresis 261 thresholding is a segmentation method requiring two thresholds. With an upper 262 threshold, seed regions definitively belonging to roots are determined. The upper 263 threshold $\left(\mathrm{t}_{\text {hys }}^{\text {high }}\right.$ ) is less relevant for segmentation accuracy and can be set a priori.

264 From the upper threshold, a region growing process connects all voxels brighter than a 265 lower threshold $\left(\mathrm{t}_{\text {hys }}^{\text {low }}\right)$. This region growing process improves edge continuity in gradient 266 images [26] and the class assignment of partial volume voxels [27], thereby reducing 267 the presence of false positives.

268 In this work, we introduce a new method to estimate the lower threshold applied 269 during hysteresis thresholding. This estimation is based on the measurement of root 270 diameters present in the image to segment. The link between the lower threshold 271 applied during hysteresis thresholding and the root diameters has been made by 272 analyzing carefully the results of the tubeness filter for increasing $\sigma$ values applied on 273 the same image of a hypothetical root (Fig. 4a). This was achieved with the following 274 sequence of operations. First, a root of a diameter $d_{r}$ is created by drawing a white 275 cylinder on a black background. Then, this root image is filtered with tubeness of 
276 increasing $\sigma$ values. Note that the absolute value of the tubeness intensity depends on

277 the gradient magnitude and hence the level of smoothing. Therefore, the contrast in the

278 tubeness filter results were normalized, i.e. the highest grey value after filtering is set to

279255 during conversion from 32-bit to 8-bit. In order to generalize the obtained results,

280 we introduce the normalized smoothing strength q which is equal to

$$
\mathrm{q}=\frac{\sigma}{\mathrm{d}_{\mathrm{r}}}
$$

282 where $\sigma$ is the smoothing strength of the tubeness filter and $d_{r}$ is the root diameter. Both

283 parameters are expressed in number of voxels. For each q value, a grey value transect

284 along the root diameter axis is plotted (Fig. 4b). It is shown that, for low q values, the

285 filtered root appears hollow and has symmetrical grey value peaks on both sides of the

286 root diametrical axis. For q values greater than 0.125 , the grey value transects have a

287 concave parabolic shape with their symmetrical axis centered on the root diametrical

288 axis. For a given q value, the grey value at the intersection of the parabola and the

289 original root outline (i.e. the vertical blue dashed lines in Fig. 4a and 4b) corresponds to

290 the optimal lower threshold $\left(\mathrm{t}_{\text {hys }}^{\text {opt }}\right)$ to use during hysteresis thresholding in order to

291 precisely capture the original root diameter. To formalize the calculation of $\mathrm{t}_{\text {hys }}^{\text {opt }}$, we

292 retrieved $\mathrm{t}_{\text {hys }}^{\text {opt }}$ (i.e. the colored dots in Fig. 4b) for all $\mathrm{q}$ values and fitted a regression

293 model (Fig. 4c) which describes best the relationship between those two parameters

294 (i.e. highest possible $\mathrm{R}^{2}$ values). We then calculated $\mathrm{t}_{\text {hys }}^{\text {opt }}$ for $\mathrm{q}=0.5$ using the model

295 regression (i.e. $\mathrm{t}_{\text {hys }}^{\text {opt }}=79$, see the dashed line in Fig. 4c). With the optimal lower 296 threshold calculated and with a measurement of the diameter of the root to segment, 
297 the sigma value of the tubeness filter can be estimated (Eq. 5). It is important to note 298 that, in case of image rescaling, the resolution factor $\left(\mathrm{f}_{\mathrm{s}}\right)$ needs to be accounted for in 299 the determination of $d_{r}$. Thus, Eq. 4 is recast to:

$$
\sigma_{\mathrm{i}}=\mathrm{q} \cdot \mathrm{d}_{\mathrm{r}, \mathrm{i}} \cdot \mathrm{f}_{s}
$$

301 where $\sigma_{\mathrm{i}}$ is the smoothing strength of the tubeness filter to use to properly segment a 302 root of a certain diameter $d_{r, i}$, $q$ is the normalized smoothing strength and $f_{s}$ is the 303 resolution factor. For the segmentation of fine roots, we segmented the images at the 304 original resolution (i.e. $f_{s}=1$ ) whereas the coarse roots were segmented with a 305 downscaled input image (i.e. $f_{s}=0.5$ ) in order to reduce processing time without 306 considerable loss of information. To determine values for $d_{r, i}$ a priori, we adopted an 307 incremented approach to account for the continuous distribution of root diameters (Fig. 308 5). This approach requires three parameters, namely the "Minimum root diameter", the 309 "Root diameter increment" and the "Maximum root diameter". All three parameters are 310 expressed in units of number of voxels. The minimum $\left(\mathrm{d}_{\mathrm{r}, \mathrm{min}}\right)$ and maximum root 311 diameter $\left(\mathrm{d}_{\mathrm{r}, \text { max }}\right)$ were determined by measuring the diameter of the finest and biggest 312 root in the image with the "Measure" tool available in ImageJ. The root diameter

313 increment $\left(\mathrm{d}_{\mathrm{r}, \text { inc }}\right)$ parameter refers to the increment at which roots of increasing

314 diameters are detected. Here, we set a root diameter increment value equal to four 315 voxels, which yields an incremented $\sigma$ value of one at the coarse resolution according to 316 Eq. 5. With these three parameters, an incremented calculation estimates the 317 appropriate sigma values of tubeness for each resolution and scale considered and root 318 diameters targeted. After filtering with tubeness and subsequent segmentation with 
319 hysteresis thresholding, the results were combined into one image through a logical

320 "Max" operation, i.e. a voxel is assigned to the root class if it is assigned to roots in at

321 least one resolution or scale. This updated approach replaces the fixed scales and

322 manually defined $\mathrm{t}_{\text {hys }}^{\text {low }}$ for each scale in Rootine $\mathrm{v} 1$. The result of the root segmentation

323 step is in Fig. 2e.

\section{Postprocessing}

The postprocessing steps aim at removing artefacts which occurred in the course

326 of segmentation. Such artefacts may include for instance segmented particulate organic

327 matter or isolated pores whose grey values are in the same range as the one of the

328 roots. First, a 3D Median filter is applied on the segmented images in order to smoothen

329 the root surfaces. The degree of filtering is determined by the kernel size of the filter

330 which needs to be given as an input parameter. On one hand, this filtering operation is

331 favorable as it trims some oversegmentation voxels extending from the roots into the

332 surroundings. On the other hand, this trimming also causes some fine root segments to

333 be disconnected from the root system. After 3D Median filtering, the unconnected

334 objects are removed using a connectivity criterion. This operation is performed with the

335 "Keep Largest" function available in the "MorpholibJ" plugin library [28]. Prior to "Keep

336 Largest", an extra slice is added at the top of the stack to ensure the connectivity of all

337 root segments from top to bottom. This is necessary when the seed from which all roots

338 emerge is not part of the image. In the case of Zea mays, adding this step is essential

339 as it allows keeping the brace and crown roots which do not directly emerge from the 340 seed but always enter the ROI from the top. 
A new feature of Rootine v. 2 is to implement a "false negatives" recovery step.

342 This step labels and evaluates every object unconnected to the root system and test

343 whether it fulfills shape criteria which evoke typical shape of roots. Those unconnected

344 objects are either segmented clusters of pores and/or segmented particulate organic

345 matter (i.e. false positives) or root segments which were disconnected due to the

346 trimming effect of the previously applied 3D Median filter (i.e. false negatives). Here, we

347 evaluate every unconnected object based on two criteria, i.e. its "Vesselness" and its

348 size. To evaluate the vesselness, we adopt a simplified formulation of the vesselness

349 function proposed by [18] and derive a "vesselness score" of individual objects. This is

350 based on the analysis of the length of the semi-axes of fitting ellipsoids to binary objects

351 instead evaluating the Hessian matrix (i.e. the $2^{\text {nd }}$ derivative of grey values) of each

352 voxel. The semi-axes of the fitting ellipsoids are noted $\lambda_{1}, \lambda_{2}$ and $\lambda_{3}$. By convention and

353 in order to make abstraction of the local orientation of the considered object in the 3D

354 space, we pose $\lambda_{1} \leq \lambda_{2} \leq \lambda_{3}$. For every object, we then compute the following

355 geometrical ratios:

$$
R a=\frac{\lambda_{2}}{\lambda_{3}}
$$

358 The first ratio accounts for the deviation from a blob-like structure. For a blob-like object 359 (i.e. $\lambda_{1} \approx \lambda_{2} \approx \lambda_{3}$ ), $R b$ will attain high values whereas it will have low values for 360 elongated objects (i.e. $\lambda_{1} \approx \lambda_{2} \ll \lambda_{3}$ ). The second ratio is essential for distinguishing 361 between plate-like and cylinder-like structures. For a plate-like object (i.e. $\lambda_{1} \ll \lambda_{2} \approx$ 
$362 \lambda_{3}$ ), $R a$ will reach its maximum whereas it will be low for elongated objects. Based on

363 the defined ratios, we evaluate how similar an object is to a cylinder by deriving the 364 vesselness score ( $v$ ) according to Eq. 8.

$$
v=\exp \left(-R b^{2}\right) \cdot \exp \left(-R a^{2}\right)
$$

366 The vesselness score can have values ranging from 0.13 for a perfect sphere to $\approx 1$ for

367 an infinitely long cylinder. The relationship between the length of the semi-axes of the 368 fitting ellipsoids, the calculated geometrical ratios and some properties of the 369 vesselness score are illustrated in Fig. 6 for simple geometrical objects, i.e. a sphere, a 370 plate and a cylinder. In addition to the vesselness criterion, a size criterion is used in 371 order to exclude small objects which originate mostly from the noise level in the image 372 and may be chance fulfill the vesselness criterion. The size criterion is given by a single 373 value being equal to the greatest length of the semi-axes of the fitting ellipsoid, i.e. $\lambda_{3}$. In 374 practice, evaluating the unconnected objects is performed in three steps. All steps rely 375 on operations available in the "MorpholibJ" plugin library. First, a label is assigned to 376 every unconnected object via the "Connected Components Labeling" function. Second, 377 for every unconnected object, the length of the semi-axes $\lambda_{1}, \lambda_{2}$ and $\lambda_{3}$ of the fitting 378 ellipsoids are computed with the "Analyze Regions 3D" function. Third, the vesselness 379 score is calculated and assigned to each label using the "Assign Measure to Label" 380 function. An object is then considered false negative only if the following conditions are 381 met:

$$
v_{i}>t_{v} \quad \text { and } \quad \lambda_{3, i}>t_{s}
$$


383 where $v_{i}$ and $\lambda_{3, i}$ are the vesselness score and size score of the object $\mathrm{i}$ and $t_{v}$ and $t_{s}$

384 are the vesselness and the size threshold respectively. The vesselness and the size

385 threshold are input parameters which need to be given and calibrated by the user. After 386 discriminating between false positives and false negatives, the false negatives are

387 added to the connected root system whereas the false positives are discarded. Fig. 7

388 illustrates this new approach of the postprocessing scheme implemented in Rootine v.2.

389 The effect of the post processing steps is shown in the difference between Fig. $2 \mathrm{e}$ and 390 Fig. $2 f$.

391 Quantification and analysis

392 Following postprocessing, the images can be analyzed and quantified in terms of 393 root length and diameter. The quantification of root length from X-ray CT data has to be 394 preceded by a step of skeletonization which conducts a medial axis transform of the 395 segmented root image. This results in an image where all roots are reduced to a 1 pixel 396 wide object which makes the calculations of root length more reliable and faster. This is 397 achieved by sequentially applying the "Skeletonize (2D/3D)" and "Analyze Skeleton 398 (2D/3D)" methods available in the BoneJ plugin library [29] implemented in ImageJ. The 399 root recovery is assessed by plotting the root length calculated after skeletonization of 400 the segmented root system and the root length analyzed with WinRHIZO (WR). By 401 imposing a line of best fit to the relationship between both root lengths (CT and WR), 402 the root recovery and the error consistency (i.e. the slope and the coefficient of 403 determination of the line of best fit respectively) can be evaluated. The quantification of 404 the root diameter distribution is performed with the "Local Thickness" plugin available in 
405 BoneJ. This method assigns to every root voxel a value corresponding to the diameter 406 of the largest sphere which locally fits into the root. The results of the local thickness 407 images are intersected with the skeleton images with a logical "And" operation. The 408 resulting images are skeletonized root systems where each medial axis voxel contains 409 the local root diameter information. This intersection is performed in order to avoid that

410 big roots contribute to more voxels than smaller roots in the histogram. The histogram of

411 the obtained images is then computed to retrieve the root length corresponding to every

412 diameter class. Note that, even though roots were destructively sampled at two different

413 layers for six replicates, the results are shown here by pooling all replicates and all

414 layers together for each scenario. For both the root length and the root diameter

415 distribution, the root length is normalized by dividing by the volume of the soil layer and 416 the results are expressed in terms of RLD.

417 Summary of the workflow and its parameters

418 This section concludes the description of the workflow. Fig. 1 and Table 1 419 provide an overview of the steps of workflow and the tunable parameters involved to 420 obtain a segmented root system from an input grey scale data acquired with X-ray CT. 421 Table 1 also list the effect and the sensitivity of the parameters on the segmentation 422 accuracy. 


\section{Root recovery and root diameter evaluation}

425 Rootine v.2 outperformed its preceding version by an increase of the root 426 recovery up to $73 \%$ of the total root length, against $29 \%$ for Rootine v. 1 in the worse

427 case scenario (Fig. 8a). The coefficient of determination is roughly equal for both 428 algorithms (i.e. $R^{2}=0.76$ and 0.79 for Rootine v. 1 and v.2 respectively). A 2D Maximum 429 Z-Projection of a selected sample (circled in black in Fig. 8a) shows that the 430 oversegmentation is low (Fig. 8b). Detected roots appear relatively smooth and there 431 are barely any root voxels extending into their surroundings. In the 2D Maximum Z432 Projection, some root segments are disconnected from the root system. Those 433 segments are the ones added by the false negatives recovery step during 434 postprocessing. Fig. 8b also shows that the gain in root length with Rootine v.2 is mainly 435 contributed by additional fine roots (operationally defined as roots having a diameter $\leq$ $436180 \mu \mathrm{m})$. The increased fine root recovery is also reflected in the root diameter 437 distribution (Fig. 9a). On top of a higher root recovery of the fine roots, Rootine v.2 also 438 enabled to better capture the root diameter of the big roots (operationally defined as 439 roots having a diameter $\geq 900 \mu \mathrm{m}$ ) as compared to its preceding version. This can be 440 seen on Fig. 9a where Rootine v.2 agrees better with WR data for the diameter classes

441 larger than $900 \mu \mathrm{m}$ as compared to Rootine v.1. The second peak corresponding to the 442 primary roots only underestimates WR values by 4 voxels for Rootine v. 2 whereas this 443 second peak is completely absent for Rootine v.1. The better agreement of the root 444 diameters captured by Rootine v.2 can also be assessed visually by superimposing the 
445 segmented images of both algorithms and by directly comparing them with the 446 greyscale X-ray CT data (Fig. 9b).

447 The root recovery in the best case scenario amounted to $114 \%$ of the total root

448 length against $99 \%$ for Rootine v.2 and Rootine v.1 respectively (Fig. 8c). Again, the

449 coefficient of determination is roughly equal for both versions (i.e. $R^{2}=0.92$ and 0.90

450 for Rootine v.1 and v.2 respectively). Similarly as for the worse case scenario, a 2D

451 Maximum Z-Projection of the segmented roots of a selected sample (Fig. 8b) offers a

452 visual comparison of the segmentation results for both versions. Here again, the

453 oversegmentation is low as roots appear smooth and devoid of any oversegmented

454. voxels at their boundaries. The increase in root recovery is also mostly contributed by

455 the addition of fine roots. The agreement of the root diameter distribution is equally 456 good for both versions (Fig. 9c).

\section{Discussions}

\section{Segmentation accuracy}

459 In the worse case scenario, most of the roots that were missed by Rootine v.1

460 and v.2 belonged to the category of the fine roots. This was expected considering that

461 one of the main challenges of the benchmark dataset is a low image resolution as 462 compared to the small diameter of the fine maize roots. Indeed, the analysis of the 463 cumulative frequency of root diameter of the WR data shows that the fine roots 464 comprise roughly $45 \%$ of the total root length. Fine roots pose a tremendous challenge 465 due to the presence of partial volume voxels at the boundary of the roots and their 
466 surroundings. This challenge is even bigger when the contrast between the roots and

467 their surroundings is low, which was true for the worse case scenario. We attempted to

468 capture more fine roots at the original resolution by reducing the minimum root diameter

469 to two voxels (i.e. resulting in a smoothing strength of $\sigma=1$ ). However, this resulted in

470 too much oversegmentation. Still, Rootine v.2 was able to capture twice as much of the

471 fine roots as compared to Rootine v.1 in the worse case scenario. This can be attributed

472 to the background removal step during preprocessing and to the false negatives

473 recovery step performed during postprocessing. The background removal prevented the

474 presence of false positives even when segmenting the images at the original resolution.

475 In contrast, the soil heterogeneity and the low signal to noise ratio of the worse case

476 scenario forced [6] to downscale the images by a factor of 2 prior root segmentation. It

477 was argued by the authors that segmenting the images at the original resolution

478 resulted in too much oversegmentation. By applying an adequate background removal

479 operation, Rootine v.2 was able to avoid oversegmentation while improving the recovery 480 of fine roots considerably. On top of an adequate background removal, the ADT step 481 increases the grey value intensities of the roots prior tubeness filtering which is 482 favorable for their subsequent detection. The false negatives recovery step also 483 contributed to a fair amount of the root recovery. By computing the root length density 484 before and after this step, we evaluated this contribution. On average, it amounted to $48525.5 \%( \pm 1.9 \%$ standard error, $n=12)$ of the total RLD. So it explained more than half of 486 the gain in root recovery between Rootine v.1 (29\%) and v.2 (73\%). 
In the best case scenario, the RLD inferred from X-ray CT was higher than the

488 one measured with WinRHIZO. This could be an indication of the presence of false

489 positives and/or oversegmentation at the boundaries of root voxels. We rule out the

490 latter since the visual inspection of the images showed virtually no root voxels extending

491 into their surroundings. The overestimation of root length with Rootine v.2 could be due

492 to the false negatives recovery step during which some actual false "positives" were

493 considered as being false "negatives" and added back to the root system. For the best

494 case scenario, the contribution of this step to the total RLD was lower than for the worse

495 case scenario and amounted to $12.5 \%$ ( $\pm 0.8 \%$ standard error, $n=12)$. The

496 overestimation of the root recovery could also be due to the uncertainties associated

497 with the root washing procedure and further analysis with WR. The Z-Projection shown

498 in Fig. 8d is one of the samples whose X-ray CT RLD data point exceeded the RLD

499 data measured with WinRHIZO (i.e. the sample circled in black above the 1:1 line in Fig.

500 8c). When taking a closer look at Fig. 8d, it is obvious that the increase in root recovery

501 is due to the addition of real roots. During root washing, a soil sample is placed on a

502 sieve, the soil is then washed off with water and the roots remaining on the sieve (here

503 having a $1 \mathrm{~mm}$ mesh size) are picked with a tweezer and stored in ethanol prior to

504 analysis. Some fine roots can easily go unnoticed on the sieve due to their size. The

505 fact that the overestimation of WR data by Rootine v.2 occurs specifically for the fine

506 roots is an indication supporting this argument. On top of the root washing, errors in WR

507 data might be induced by an uneven distribution of the roots on the tray used for

508 scanning. An uneven distribution of the roots might cause two fine roots located very

509 close to each other to be detected as one root with a larger diameter instead. During the 
510 analysis with the WinRHIZO software, a noise threshold value has to be set to exclude

511 small dirt particles from the root length calculation. A high noise threshold value leads to

512 smooth root surfaces but also results in the loss of fine roots. An improper setting of this

513 parameter may then also induce errors. We rule out the effect of storing roots in ethanol

514 on the WR results as this procedure has proved to be valid to conserve root samples

515 without considerable influence on the measurements of root length (i.e. $<1 \%$ of

516 underestimation) and diameter (i.e. $5 \%$ of underestimation) [30]. Both the potential loss

517 of roots during washing and the underestimated capture of fine roots by WinRHIZO

518 could explain the overestimation of the root recovery in the best case scenario. Note

519 that if the RLD data characterized with WR is underestimated, it is likely that the root

520 recovery in the worse case scenario is overestimated.

521 The segmentation accuracy was evaluated based on quantitative aspects such

522 as the root recovery and the comparison of root diameter distribution. Additionally,

523 segmentation accuracy was also evaluated visually based on qualitative aspects, i.e.

524 how accurately the root diameter outlines were segmented. With Rootine v.1, the

525 primary roots often showed irregular shapes. With Rootine v.2, primary roots were

526 segmented with a higher accuracy and showed a prominent circular shape when viewed

527 in a 2D x-y cross section. This can be seen in Fig. 9b and 9d. This increase in accuracy

528 can most likely be ascribed to the fact that more scales were considered during the

529 tubeness filtering at the coarse resolution with Rootine v.2. However, this difference in

530 capturing the root diameter outlines was not big enough to be reflected in the root

531 diameter distribution in the best case scenario. By comparing the results of Rootine v.1 
532 and v.2 visually, it was also noticeable that the latest version showed less false

533 positives and less segmentation artefacts. Such segmentation artefacts include for

534 instance the oversegmentation of roots growing along the plastic wall of the pot. Rootine

535 v.1 did not feature a pot wall detection and removal step. Since the root average grey

536 value is close to the one of the pot wall (see the corresponding grey values of $\bar{v}_{r}$ and P1

537 in Fig. 3b), it was often observed on the results of Rootine v.1 that segmented root were

538 extending into the plastic wall. This was particularly true in the best case scenario.

539 Numbers of tunable parameters

540 One of the objectives of this work was to develop an algorithm in which the

541 numbers of tunable parameters is reduced. Note that we consider as "tunable" the

542 parameters which require adjustments and calibration when applied to other datasets

543 (i.e. experiments with different plants, scan settings and/or soil heterogeneity).

$544 \quad$ In Rootine v.1, we identified twelve tunable parameters in total, namely five for

545 the preprocessing, six for the root segmentation and one for the postprocessing. For the

546 preprocessing, one parameter was used for filtering the original greyscale image (i.e.

547 the contrast threshold of the 3D NLM filter), two for edge enhancement (i.e. the blur

548 radius and the mask weight of the Unsharp mask filter) and two for masking the pores

549 by single thresholding (i.e. pores were masked with different thresholds at the original

550 and at the coarse resolution). For the root segmentation step at the original resolution,

551 one $\sigma$ value and the corresponding $\mathrm{t}_{\text {hys }}^{\text {low }}$ for hysteresis thresholding method were used.

552 For the root segmentation step at the coarse resolution, three $\sigma$ values were used. The

553 obtained results of the tubeness filtering were then merged and one $\mathrm{t}_{\text {hys }}^{\text {low }}$ for the 
554 hysteresis thresholding method was used to segment the results of the coarse root

555 detection (this amounts to four parameters). The upper threshold for hysteresis

556 thresholding $\left(\mathrm{t}_{\text {hys }}^{\text {high }}\right.$ ) was kept constant and high enough for every tubeness filtering

557 scale and is thus considered non-tunable. For the postprocessing, Rootine v.1 relied on

558 one parameter, i.e. the kernel size of the 3D Median filter.

559 In Rootine v.2, the pore masking thresholds used by [6] were replaced by the

560 parameters the root grey value faction $\left(f_{r}\right)$ and the root grey value range $\left(R_{r}\right)$. The

561 parameters of the 3D NLM filter and the Unsharp mask filtering are used in the new

562 version. With the introduction of the automatic calculation of the $\sigma$ values and $\mathrm{t}_{\text {hys }}^{\text {opt }}$ and

563 keeping $t_{h y s}^{\text {high }}$ high enough and constant for every tubeness filtering scale, the number

564 of parameters required for the root segmentation was reduced to two (i.e. $d_{r, \min }, d_{r, \max }$ ).

565 As they have been set once for the new root segmentation approach, we consider the

566 parameters $\mathrm{q}, \mathrm{f}_{s}$ and $\mathrm{d}_{r, \text { inc }}$ as "quasi-fixed" and therefore non-tunable. For the

567 postprocessing, a smoothening step of the root outline was performed with the "3D

568 Median" filtering step requiring one parameter (i.e. the kernel size). The false negatives

569 recovery step added two parameters, i.e. the vesselness $\left(t_{v}\right)$ and the size $\left(t_{s}\right)$

570 thresholds. In total, the number of tunable parameters in Rootine v.2 was reduced to

571 ten, i.e. five for the preprocessing of the image, two for the root segmentation and three

572 for the postprocessing. It is worth noting that more parameters are required to use the

573 full functionalities of Rootine v.2. Such parameters include for instance the coordinates

574 of the ROI mask. Those coordinates need to be directly evaluated on the image and are

575 not considered to influence the segmentation results if appropriate values are given. 


\section{Runtime and overall usability of Rootine v.1 and v.2}

577 Besides the segmentation accuracy and the number of parameters, the

578 assessment of the performance of a segmentation algorithm also has to take into

579 account the time it takes to process the images. This is important for application in high-

580 throughput root phenotyping based on X-ray CT data analysis. To process a stack

581 having a dimension of $1750 \times 1750 \times 3000$ voxels (in $x, y$ and $z$ dimension, size $\approx 8.6 \mathrm{~GB}$ ),

582 it took Rootine v.1 3.8 hours to complete the preprocessing, the segmentation and the 583 postprocessing steps. In comparison, Rootine v.2 took 6.8 hours to complete the same

584 steps. The second version is 1.8 times slower than the first version for the analyzed

585 image size. This is mainly due to the consideration of more scales during tubeness

586 filtering at the coarse resolution. For both algorithms, the evaluation of the runtime was

587 performed on a workstation having 64 Intel® Xeon® Gold 6142 cores running at 2.60

$588 \mathrm{GHz}$ each. To this date, filtering with the tubeness filter represents the bottleneck of the

589 workflow. This is related to the fact that the tubeness filter is only implemented in a

590 single threaded fashion in the ImageJ software. There should be no fundamental

591 constraint that would restrict its parallelisation and Rootine (regardless of the versions)

592 would benefit a lot from it. Note that there exists a multithreaded implementation of the

593 tubeness plugin which was developed in the context of ImageJ Ops [31]. We have

594 however not tested it. It is worth noting that both algorithms can be run in a user

595 interaction-free mode (i.e. from the command line) once the parameters are adjusted.

596 This provides an advantage and, in our opinion, reduces the necessity of having a fast

597 algorithm as the macro can run in the background and/or overnight. When it comes to

598 RSA studies, a longer runtime can be well accepted as long as the root recovery is 
599 substantially increased. Despite the longer time required to segment images, we

600 assume that the increase of the overall usability of Rootine v.2 can save the user some

601 time for the adjustments of the parameters. Indeed, Rootine v.2 features input

602 parameters which are linked to root physiological properties such as their grey value

603 and their diameter. These tunable parameters are easy to adjust as they can be directly

604 assessed visually on a test image.

\section{Conclusion}

606 Rootine v.2 has been developed for improved root segmentation accuracy in X-ray CT

607 data. It exploits intrinsic properties of root systems such as the connectivity of root

608 branches and the cylindrical shape of roots to distinguish roots from the background. It

609 was demonstrated that Rootine v.2 outperforms its precursor version in terms of root

610 recovery, which was already proved to be superior to other state-of-the-art root

611 segmentation methods. The gain in root recovery could be mainly ascribed to the

612 absolute difference transform of the greyscale data prior to shape detection with a

613 series of tubeness filters and to a false negatives recovery step. The other major

614 advancements of Rootine v.2 are (1) a new pot wall detection and removal step, (2) a

615 calculation of the root average grey value based on a histogram analysis and (3) an

616 automatic calculation of thresholds for hysteresis thresholding of the tubeness image.

617 Moreover, the analysis of the root diameter distribution is readily integrated in the new

618 version. The total number of tunable parameters for the entire workflow was reduced

619 from twelve to ten. Rootine v.2, in comparison to Rootine v.1, functions less in a "black

620 box" fashion as its parameters can be more easily interpreted and are easier to adjust. 
621 The proposed method has the potential of improving high-throughput root phenotyping

622 procedures based on X-ray CT data analysis. Similarly as its preceding version, Rootine

623 V.2 is a macro for the image processing software Image J and is made freely available to 624 the public.

\section{Declarations}

626 Ethics approval and consent to participate

627 Not applicable

628 Consent for publication

629 Not applicable

630 Authors' contributions

631 MP and SS developed the Rootine v.2 code. MP processed and analysed the X-ray CT 632 data. MP wrote the manuscript and produced the figures with the assistance of SS. EL 633 processed the WinRHIZO data. DV facilitated the research and provided assistance 634 with the writing and edition of the manuscript. All authors read and approved the final 635 manuscript.

\section{Acknowledgement}

637 We thank Caroline Marcon and Frank Hochholdinger (University of Bonn) for providing 638 the maize B73 seeds. We also thank Maik Lukas and Sebastian R.G.A. Blaser for 639 providing useful comments on the manuscript. 


\section{Funding}

641 This project was carried out in the framework of the priority programme 2089

642 "Rhizosphere spatiotemporal organization - a key to rhizosphere functions" funded by

643 Deutsche Forschungsgemeinschaft (project number 403640293).

\section{Competing interests}

645 The authors declare that they have no competing interests.

\section{Data availability}

647 The Rootine v.2 workflow is available as supporting information. The CT images (top

648 and bottom, approx. 4GB each) of one pot of the worse case scenario are provided

649 through the file link: https://nc.ufz.de/s/MyPPgjFJZe6nNxK (password: Rootinev2). for

650 testing reproducibility. The temporary file link will be replaced by a permanent archive

651 link after manuscript acceptance. The entire datasets used during the current study are

652 available from the corresponding author on reasonable request.

653

654

655 


\section{Tables}

657 Table 1 Summary of the parameters, their values, their effects and their sensitivity

658 on the segmentation accuracy.

\begin{tabular}{|c|c|c|c|c|c|}
\hline \multirow[t]{2}{*}{ Step } & \multirow[t]{2}{*}{ Parameter } & \multicolumn{2}{|c|}{ Value } & \multirow[t]{2}{*}{ Effect } & \multirow{2}{*}{$\begin{array}{c}\text { Sensitivity on } \\
\text { the } \\
\text { segmentation } \\
\text { accuracy }\end{array}$} \\
\hline & & $\begin{array}{l}\text { Worse } \\
\text { case }\end{array}$ & $\begin{array}{l}\text { Best } \\
\text { case }\end{array}$ & & \\
\hline Image filtering & $\begin{array}{l}\text { Contrast } \\
\text { threshold } \\
\left(\mathrm{t}_{\text {con }}\right)\end{array}$ & 60 & 60 & $\begin{array}{l}\text { Controls the degree of } \\
\text { smoothening (i.e. noise } \\
\text { removal) of the input image. }\end{array}$ & Medium \\
\hline \multirow{2}{*}{$\begin{array}{c}\text { Edge } \\
\text { enhancement }\end{array}$} & Blur radius & 1 & 0.9 & \multirow{2}{*}{$\begin{array}{l}\text { Both parameters control the } \\
\text { degree of sharpening of the } \\
\text { image, i.e. increase the } \\
\text { contrast at the boundaries } \\
\text { between roots and pores } \\
\text { and soil matrix. }\end{array}$} & \multirow{2}{*}{ High } \\
\hline & Mask weight & 0.7 & 0.8 & & \\
\hline \multirow[b]{2}{*}{$\begin{array}{l}\text { Background } \\
\text { removal }\end{array}$} & $\begin{array}{l}\text { Root grey } \\
\text { value factor } \\
\quad\left(\mathrm{f}_{r}\right)\end{array}$ & 0.10 & 0.18 & $\begin{array}{l}\text { Sets the grey value of the } \\
\text { roots. }\end{array}$ & Very high \\
\hline & $\begin{array}{l}\text { Root grey } \\
\text { value range } \\
\left(\mathrm{R}_{r}\right)\end{array}$ & 65 & 70 & $\begin{array}{l}\text { Controls the root grey value } \\
\text { window around the root } \\
\text { grey value. If set too high, } \\
\text { overestimation of roots into } \\
\text { their surroundings will } \\
\text { occur. If set too low, loss of } \\
\text { roots will occur. }\end{array}$ & Very high \\
\hline $\begin{array}{l}\text { Detect fine } \\
\text { roots }\end{array}$ & $\begin{array}{l}\text { Minimum root } \\
\text { diameter } \\
\left(\mathrm{d}_{r, \min }\right)\end{array}$ & 4 & 4 & $\begin{array}{l}\text { Controls the root recovery } \\
\text { of the fine roots. If set too } \\
\text { high, the fine roots are not } \\
\text { detected. If set too low, } \\
\text { oversegmentation may } \\
\text { occur depending on the } \\
\text { noise level of the image. }\end{array}$ & High \\
\hline $\begin{array}{l}\text { Detect coarse } \\
\text { roots }\end{array}$ & $\begin{array}{l}\text { Maximum root } \\
\text { diameter } \\
\left(\mathrm{d}_{r, \max }\right)\end{array}$ & 28 & 28 & $\begin{array}{l}\text { Controls the accuracy of } \\
\text { the root diameter outline of } \\
\text { the biggest root. If set too } \\
\text { high, the diameter of the } \\
\text { biggest root is } \\
\text { overestimated and } \\
\text { computational time is } \\
\text { wasted. If set too low, the } \\
\text { diameter of the biggest root }\end{array}$ & Medium \\
\hline
\end{tabular}




\begin{tabular}{|c|c|c|c|c|c|}
\hline & & & & is underestimated. & \\
\hline $\begin{array}{c}\text { False positives } \\
\text { removal }\end{array}$ & $\begin{array}{l}\text { Kernel size of } \\
\text { median filter }\end{array}$ & 3 & 2 & $\begin{array}{l}\text { Controls the degree of } \\
\text { smoothening of the roots } \\
\text { and trimming of over } \\
\text { segmented voxels. If set } \\
\text { too high, root loss occurs } \\
\text { whereas low values result } \\
\text { in the presence of many } \\
\text { false positives. }\end{array}$ & High \\
\hline \multirow{2}{*}{$\begin{array}{l}\text { False } \\
\text { negatives } \\
\text { recovery }\end{array}$} & $\begin{array}{l}\text { Size } \\
\text { threshold } \\
\quad\left(t_{s}\right)\end{array}$ & 25 & 25 & \multirow{2}{*}{$\begin{array}{l}\text { Both parameters control the } \\
\text { quality of the false } \\
\text { negatives added in the root } \\
\text { system. If set too low, many } \\
\text { false positives are } \\
\text { considered negatives, } \\
\text { adding noise to the results. } \\
\text { If set too high, root loss } \\
\text { occurs. }\end{array}$} & High \\
\hline & $\begin{array}{l}\text { Vesselness } \\
\text { threshold } \\
\left(\mathrm{t}_{v}\right)\end{array}$ & 0.85 & 0.9 & & High \\
\hline
\end{tabular}

661 1. Mooney SJ, Pridmore TP, Helliwell J, Bennett MJ: Developing X-ray computed 662 tomography to non-invasively image 3-D root systems architecture in soil.

$663 \quad$ Plant and soil 2012, 352:1-22.

664 2. Blaser SR, Koebernick N, Spott O, Thiel E, Vetterlein D: Dynamics of localised 665 nitrogen supply and relevance for root growth of Vicia faba ('Fuego') and 666 Hordeum vulgare ('Marthe') in soil. Scientific Reports 2020, 10:1-16.

667 3. Blaser SR, Schlüter S, Vetterlein D: How much is too much?-Influence of X668 ray dose on root growth of faba bean (Vicia faba) and barley (Hordeum $669 \quad$ vulgare). PloS one 2018, 13:e0193669. 
670 4. Flavel RJ, Guppy CN, Rabbi SM, Young IM: An image processing and 671 analysis tool for identifying and analysing complex plant root systems in 672 3D soil using non-destructive analysis: Root1. PloS one 2017, 12:e0176433.

673 5. Flavel RJ, Guppy CN, Tighe M, Watt M, McNeill A, Young IM: Non-destructive 674 quantification of cereal roots in soil using high-resolution X-ray 675 tomography. Journal of Experimental Botany 2012, 63:2503-2511.

676 6. Gao W, Schlüter S, Blaser SR, Shen J, Vetterlein D: A shape-based method for 677 automatic and rapid segmentation of roots in soil from X-ray computed 678 tomography images: Rootine. Plant and Soil 2019, 441:643-655.

679 7. Koebernick N, Weller U, Huber K, Schlüter S, Vogel H-J, Jahn R, Vereecken H, 680 Vetterlein D: In Situ Visualization and Quantification of Three-Dimensional 681 Root System Architecture and Growth Using X-Ray Computed 682 Tomography. Vadose Zone Journal 2014, 13:1-10.

683 8. Kuka K, Illerhaus B, Fox CA, Joschko M: X-ray Computed Microtomography 684 for the Study of the Soil-Root Relationship in Grassland Soils. Vadose Zone 685 Journal 2013, 12:1-10.

686 9. Maenhout P, Sleutel S, Xu H, Van Hoorebeke L, Cnudde V, De Neve S: Semi687 automated segmentation and visualization of complex undisturbed root 688 systems with X-ray $\mu \mathrm{CT}$. Soil and Tillage Research 2019, 192:59-65.

689 10. Mairhofer S, Zappala S, Tracy SR, Sturrock C, Bennett M, Mooney SJ, Pridmore 690 T: RooTrak: automated recovery of three-dimensional plant root 691 692 architecture in soil from X-ray microcomputed tomography images using visual tracking. Plant physiology 2012, 158:561-569. 
693 11. Metzner R, Eggert A, Van Dusschoten D, Pflugfelder D, Gerth S, Schurr U, 694 Uhlmann N, Jahnke S: Direct comparison of MRI and X-ray CT technologies for 3D imaging of root systems in soil: potential and challenges for root trait quantification. Plant methods 2015, 11:1-11.

697 12. Pfeifer J, Kirchgessner N, Colombi T, Walter A: Rapid phenotyping of crop root systems in undisturbed field soils using X-ray computed tomography.

13. Soltaninejad M, Sturrock CJ, Griffiths M, Pridmore TP, Pound MP: Three Dimensional Root CT Segmentation using Multi-Resolution EncoderDecoder Networks. IEEE Transactions on Image Processing 2020, 29:66676679.

704 14. Tabb A, Duncan KE, Topp CN: Segmenting root systems in X-ray computed 705 tomography images using level sets. In 2018 IEEE Winter Conference on

15. Teramoto S, Takayasu S, Kitomi Y, Arai-Sanoh Y, Tanabata T, Uga Y: High708

709 throughput three-dimensional visualization of root system architecture of rice using X-ray computed tomography. Plant Methods 2020, 16:1-14.

711

712 Applications of Computer Vision (WACV). IEEE; 2018: 586-595.

17. Schlüter S, Sheppard A, Brown K, Wildenschild D: Image processing of multiphase images obtained via X-ray microtomography: a review. Water Resources Research 2014, 50:3615-3639. 
716 18. Frangi AF, Niessen WJ, Vincken KL, Viergever MA: Multiscale vessel

717 enhancement filtering. In International conference on medical image computing

718 and computer-assisted intervention. Springer; 1998: 130-137.

719 19. Schulz H, Postma JA, van Dusschoten D, Scharr H, Behnke S: Plant root 720 system analysis from MRI images. In Computer Vision, Imaging and Computer

721 Graphics Theory and Application. Springer; 2013: 411-425

722 20. Schindelin J, Arganda-Carreras I, Frise E, Kaynig V, Longair M, Pietzsch T,

723 Preibisch S, Rueden C, Saalfeld S, Schmid B: Fiji: an open-source platform 724 for biological-image analysis. Nature methods 2012, 9:676-682.

725 21. Biot E, Crowell E, Hofte H, Maurin Y, Vernhettes S, Andrey P: A new filter for 726 spot extraction in $\mathbf{N}$-dimensional biological imaging. In 2008 5th IEEE International Symposium on Biomedical Imaging: From Nano to Macro. IEEE; 2008: 975-978.

729 22. Tristán-Vega A, García-Pérez V, Aja-Fernández S, Westin C-F: Efficient and 730 robust nonlocal means denoising of MR data based on salient features matching. Computer methods and programs in biomedicine 2012, 105:131-144.

732 23. Ibanez L, Schroeder W, $\mathrm{Ng} \mathrm{L}$, Cates J: The ITK software guide: updated for 733 ITK version 2.4. Kitware New York, NY; 2005.

734 24. Sheppard AP, Sok RM, Averdunk H: Techniques for image enhancement and 735 segmentation of tomographic images of porous materials. Physica A: $736 \quad$ Statistical mechanics and its applications 2004, 339:145-151. 
737 25. Ollion J, Cochennec J, Loll F, Escudé C, Boudier T: TANGO: a generic tool for

738 high-throughput 3D image analysis for studying nuclear organization.

739 Bioinformatics 2013, 29:1840-1841.

740 26. Canny J: A computational approach to edge detection. IEEE Transactions on $741 \quad$ pattern analysis and machine intelligence 1986:679-698.

742 27. Schlüter S, Weller U, Vogel H-J: Segmentation of X-ray microtomography 743 images of soil using gradient masks. Computers \& Geosciences 2010, $744 \quad 36: 1246-1251$.

745 28. Legland D, Arganda-Carreras I, Andrey P: MorphoLibJ: integrated library and 746 plugins for mathematical morphology with ImageJ. Bioinformatics 2016, $747 \quad 32: 3532-3534$.

748 29. Doube M, Kłosowski MM, Arganda-Carreras I, Cordelières FP, Dougherty RP, 749 Jackson JS, Schmid B, Hutchinson JR, Shefelbine SJ: BoneJ: free and $750 \quad$ extensible bone image analysis in ImageJ. Bone 2010, 47:1076-1079.

751 30. Logsdon S, Reneau R: Influence of storage methods on corn root length 752 measurements. Plant and soil 1988, 111:155-157.

753 31. Tinevez, JY: The Tubeness filter: enhance filamentous structures of a 754 specified thickness [https://github.com/imagej/imagej-ops/pull/527]. Accessed 755 on 21 Oct 2020.

\section{Figure legends}

757 Fig. 1 Synoptic view of the Rootine v.2 workflow including the comparison with 758 Rootine v.1. 
759 Fig. 2 Results of the steps of Rootine v.2 for a subvolume of the worse case

760 scenario; (a) The original greyscale image; (b) The obtained image after denoising with

761 the 3D NLM filter; (c) The obtained image after performing edge enhancement of

762 subfigure b; (d) Resulting image after background removal via an absolute difference

763 transform on subfigure c; (e) Results of the root segmentation applied on subfigure d

764 before applying postprocessing steps; (f) Segmented roots after applying the

765 postprocessing steps on subfigure e.

766 Fig. 3 Mask creation and calculation of the average root grey value based on

767 characteristic peaks. Subfigure (a) depicts the drawing of a circular ROI bounded

768 within the pot wall (red circle) on a 2D section of the worse case scenario. The bounded

$769 \mathrm{ROI}$ serves the purpose of creating a mask. By extension of this bounded ROI by 50

770 pixels, an extended $\mathrm{ROI}$ is created (blue line); (b) Histogram of the bounded and

771 extended ROI illustrated in subfigure a. The extended ROI serves the purpose of

772 creating a peak in the histogram which will be used to calculate the average root grey

773 value.

774 Fig. 4 Estimation of $\sigma$ values of the tubeness filters and the optimal lower

775 thresholds of hysteresis thresholding. (a) Results of the tubeness filter on a

776 hypothetical root of a diameter $d_{r}$ obtained for different $q$ values. The dashed blue lines

777 show the original root outline whereas the solid yellow lines show the position of the

778 transects used to plot the grey values along the root diameter axis; (b) Transects of the

779 grey values along the root diameter axis for some of the q values shown in subfigure a.

780 The color dots at the intersection between the root outline and the grey value parabola 
781 correspond to $t_{h y s}^{\text {opt }}$ for a given q value; (c) Line of best fit imposed on the couple of

782 points $\mathrm{q}$ and $\mathrm{t}_{\text {hys }}^{\text {opt }}$. In this study, we calculated $\mathrm{t}_{\text {hys }}^{\text {opt }}$ corresponding to $\mathrm{q}=0.5$ using the

783 model regression. The calculated value is indicated by the dashed line (i.e. $\mathrm{t}_{\text {hys }}^{\text {opt }}=79$ ).

784 Fig. 5 Approach for the detection of roots of increasing diameters at the fine and 785 coarse resolutions.

786 Fig. 6 Vesselness score, $\mathrm{Rb}$ and $\mathrm{Ra}$ values for a sphere, a plate and a cylinder.

787 Fig. 7 Illustration of the postprocessing steps implemented in Rootine v.2. First, a

788 3D Median filter is applied on the results of the root segmentation step. Then, all 789 connected objects are kept by applying the "Keep Largest" function. In order to ensure

790 full connectivity of the roots at the top of the stack, a slice is added at the top (left-hand 791 side of the figure). The remaining unconnected objects are subject to a test evaluating 792 their shape, i.e. their "vesselness" and size. This is illustrated here by showing a Z-

793 Projection of a $400 \times 400 \times 400$ image from the best case scenario dataset (right-hand 794 side of the figure). The green scale bar indicates the vesselness score whereas the red 795 scale bar indicates the size score. The intensity of the yellow color depicts the 796 combination of these two scores. If an object meets both the vesselness and size 797 threshold, it is considered as a false negative and will subsequently be added to the 798 connected root system. If not, it will be considered as a false positive and will thereafter 799 be discarded.

800 Fig. 8 Root recovery of Rootine v.2 for the worse and best case scenario. (a\&c) 801 Comparison with the former Rootine v.1 and the root length determined with destructive 
802 sampling and scanning of washed-off roots (WinRHIZO) for the worse case and the

803 best case scenario respectively. The dashed line indicates the 1:1 line. (b\&d) Visual

804 comparison of the segmented root systems obtained with Rootine v.1 and Rootine v.2

805 for the corresponding sample circled in black on subfigures a and $c$ for the worse case

806 and the best case scenario respectively. Roots detected by both algorithms are

807 depicted in black, the ones only detected by Rootine v.2 are shown in blue, whereas

808 roots only detected by Rootine v.1 are shown in red.

809 Fig. 9 Root diameter distribution and root outline accuracy for the worse and best

810 case scenario. (a\&c) Root length density distribution as a function of root diameter for

811 Rootine v.1 and v.2 and the destructive sampling data obtained by scanning washed-off

812 roots (WinRHIZO) for the worse case and the best case scenario respectively. The

813 semitransparent ribbon denotes the standard error of the measurements $(n=12)$. (b\&d)

814 Visual comparison of the segmented root diameter outlines for both Rootine v.1 and

815 Rootine v.2 supported by the original X-ray CT greyscale data for the worse case and

816 the best case scenario respectively. Roots detected by both algorithms are depicted in

817 black, the ones only detected by Rootine v.2 are shown in blue, whereas roots only

818 detected by Rootine v.1 are shown in red. Dashed horizontal black lines highlight the

819 fact that Rootine v.2 better captures root diameter in comparison with Rootine v.1. 


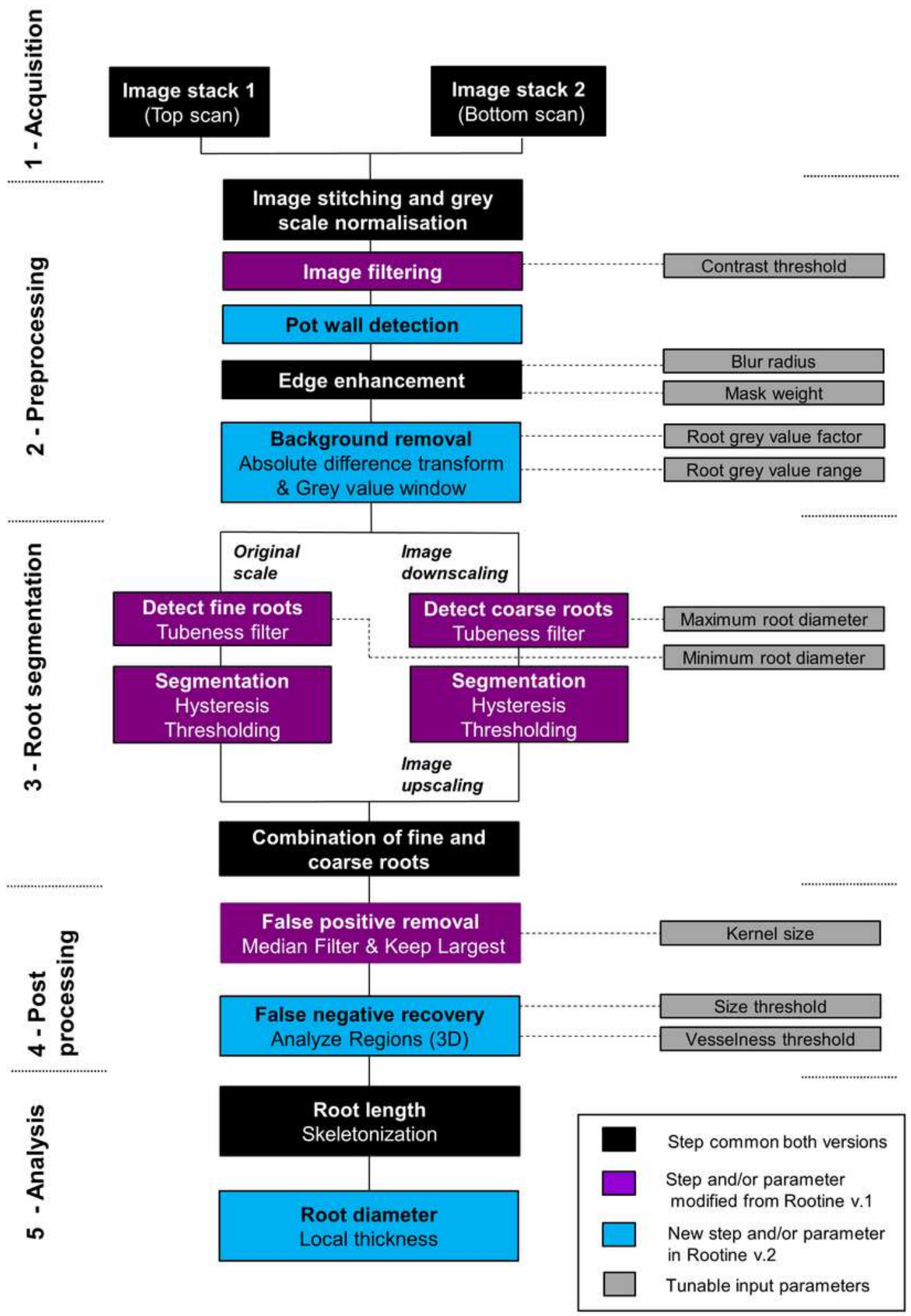

\section{Figure 1}

Synoptic view of the Rootine v.2 workflow including the comparison with Rootine v.1. 

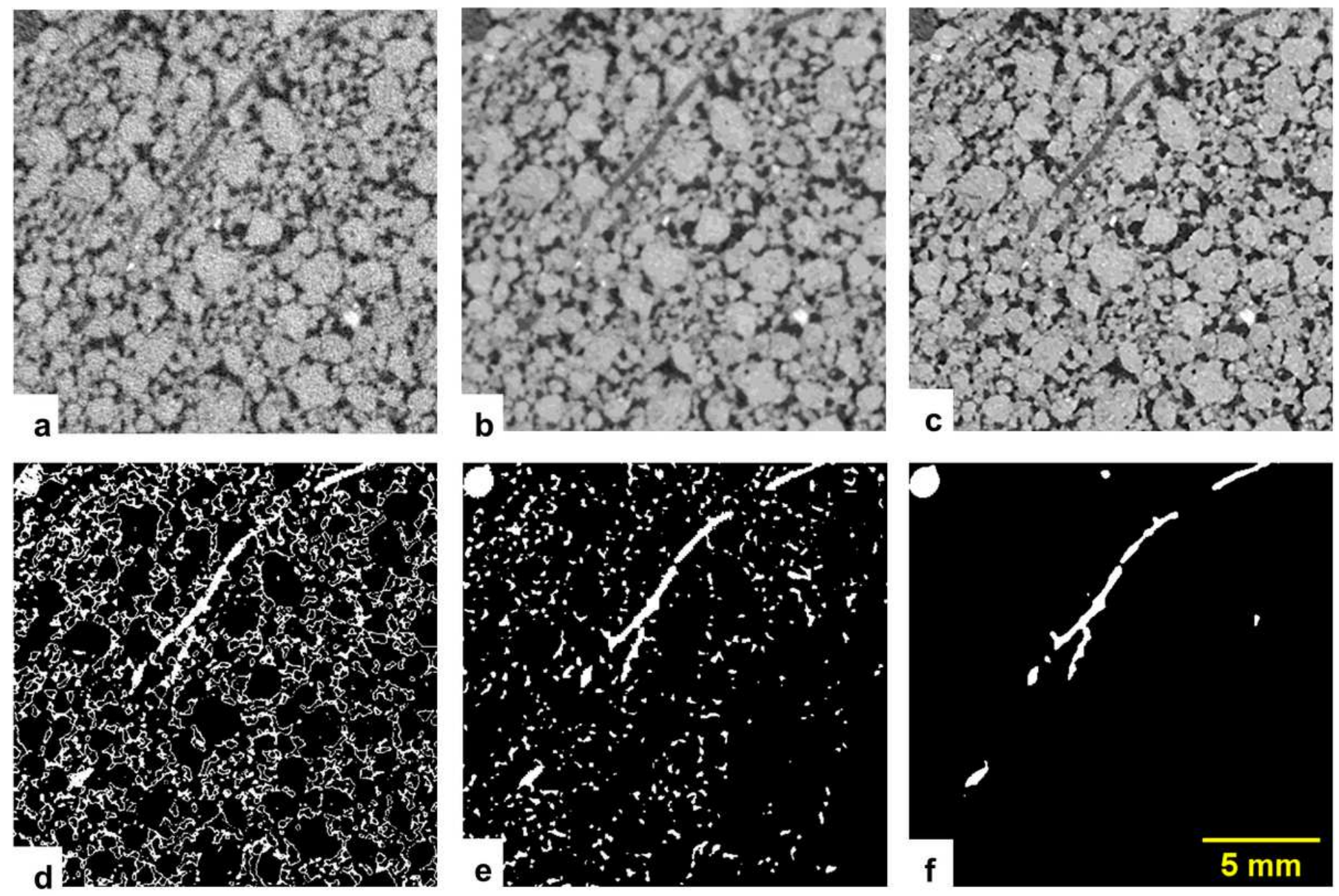

Figure 2

Results of the steps of Rootine v. 2 for a subvolume of the worse case scenario; (a) The original greyscale image; (b) The obtained image after denoising with the 3D NLM filter; (c) The obtained image after performing edge enhancement of subfigure $b$; (d) Resulting image after background removal via an absolute difference transform on subfigure $c$; (e) Results of the root segmentation applied on subfigure $d$ before applying postprocessing steps; (f) Segmented roots after applying the postprocessing steps on subfigure e. 

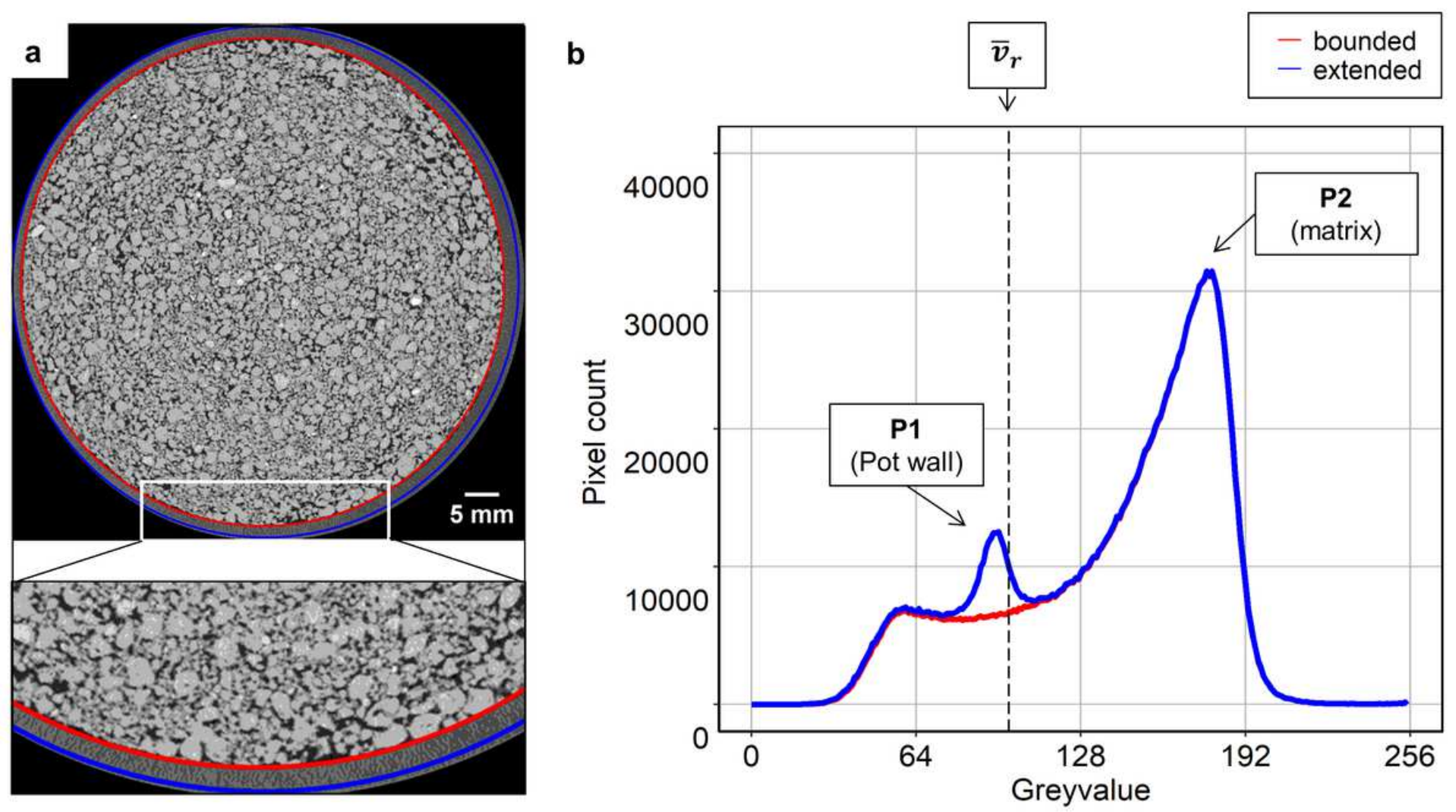

Figure 3

Mask creation and calculation of the average root grey value based on characteristic peaks. Subfigure (a) depicts the drawing of a circular ROI bounded within the pot wall (red circle) on a 2D section of the worse case scenario. The bounded ROI serves the purpose of creating a mask. By extension of this bounded ROI by 50 pixels, an extended ROI is created (blue line); (b) Histogram of the bounded and extended ROI illustrated in subfigure a. The extended ROI serves the purpose of creating a peak in the histogram which will be used to calculate the average root grey value. 


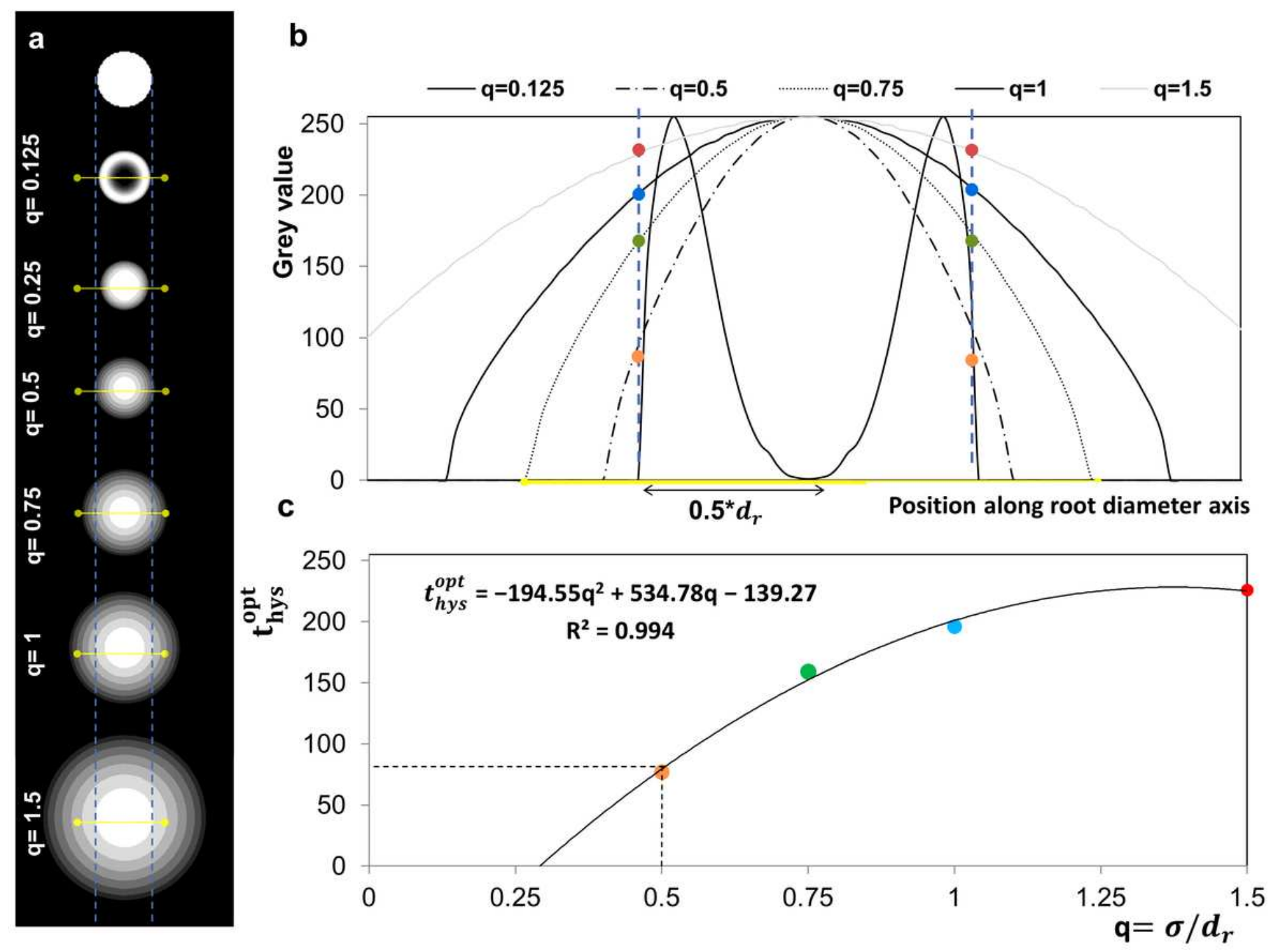

Figure 4

Estimation of $\sigma$ values of the tubeness filters and the optimal lower thresholds of hysteresis thresholding. (a) Results of the tubeness filter on a hypothetical root of a diameter dr obtained for different q values. The dashed blue lines show the original root outline whereas the solid yellow lines show the position of the transects used to plot the grey values along the root diameter axis; (b) Transects of the grey values along the root diameter axis for some of the q values shown in subfigure a. The color dots at the intersection between the root outline and the grey value parabola correspond to t_hys ${ }^{\wedge}$ opt for a given $q$ value; (c) Line of best fit imposed on the couple of points q and t_hys ${ }^{\wedge}$ opt. In this study, we calculated t_hys ${ }^{\wedge}$ opt corresponding to $\mathrm{q}=0.5$ using the model regression. The calculated value is indicated by the dashed line (i.e. t_hys ${ }^{\wedge}$ opt $=79$ ). 


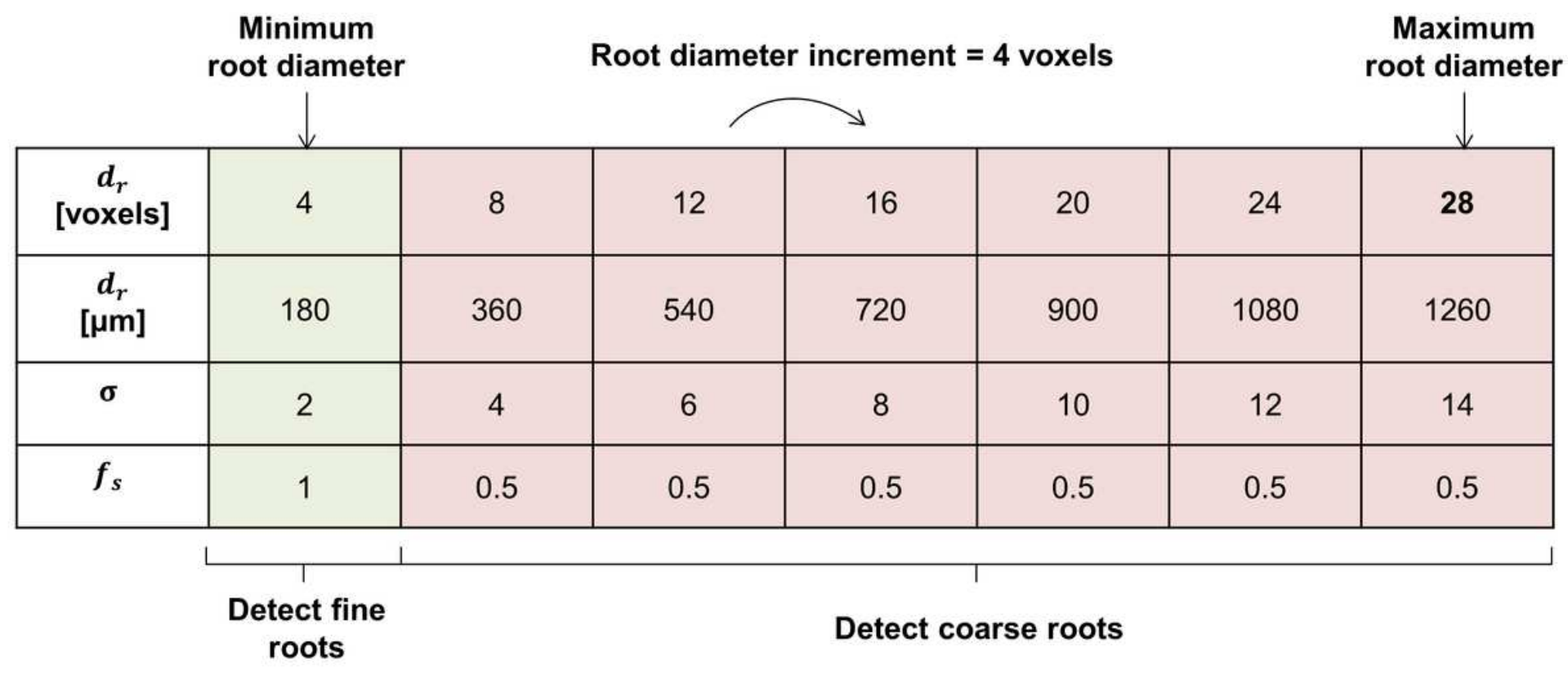

Figure 5

Approach for the detection of roots of increasing diameters at the fine and coarse resolutions.
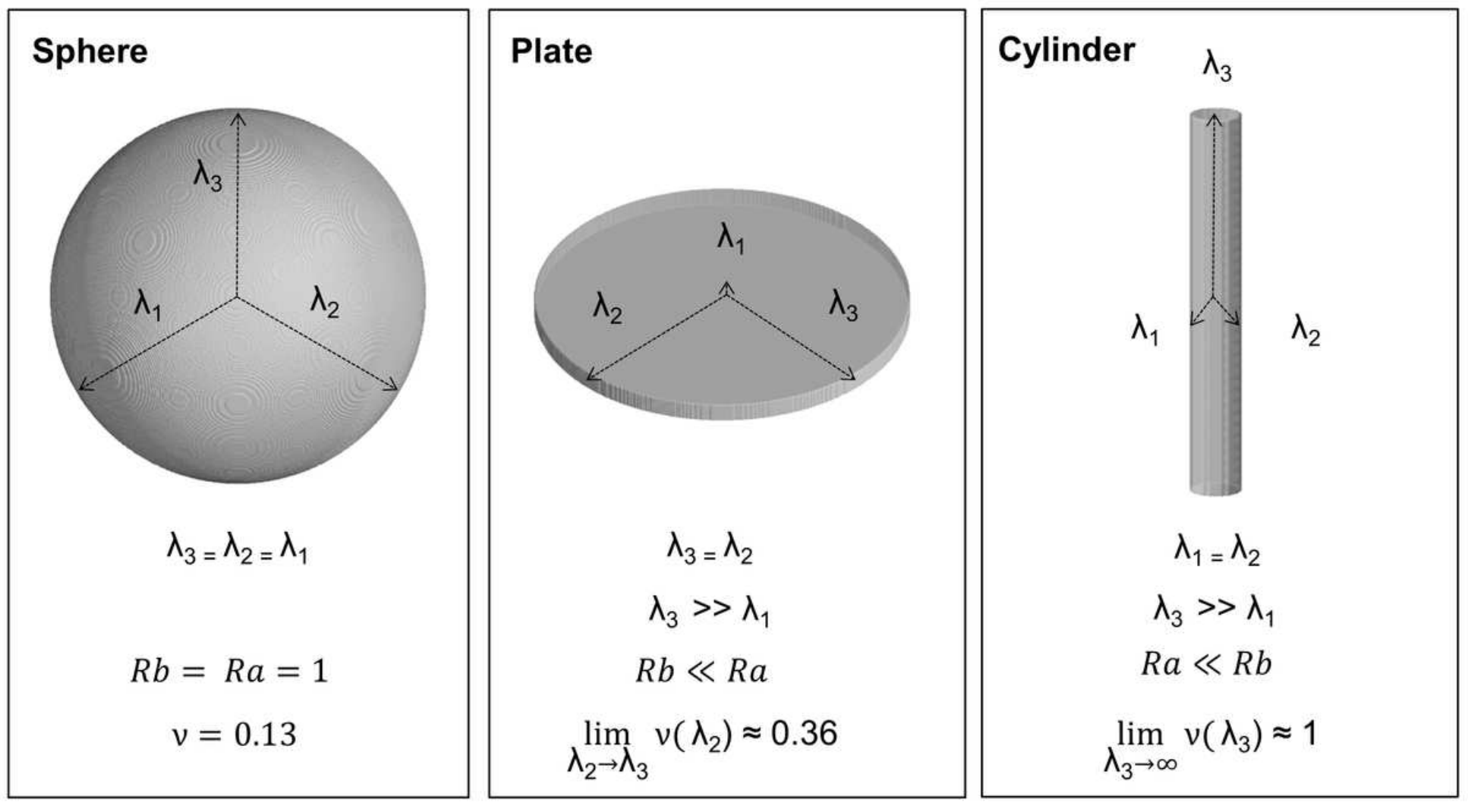

Figure 6

Vesselness score, $\mathrm{Rb}$ and $\mathrm{Ra}$ values for a sphere, a plate and a cylinder. 


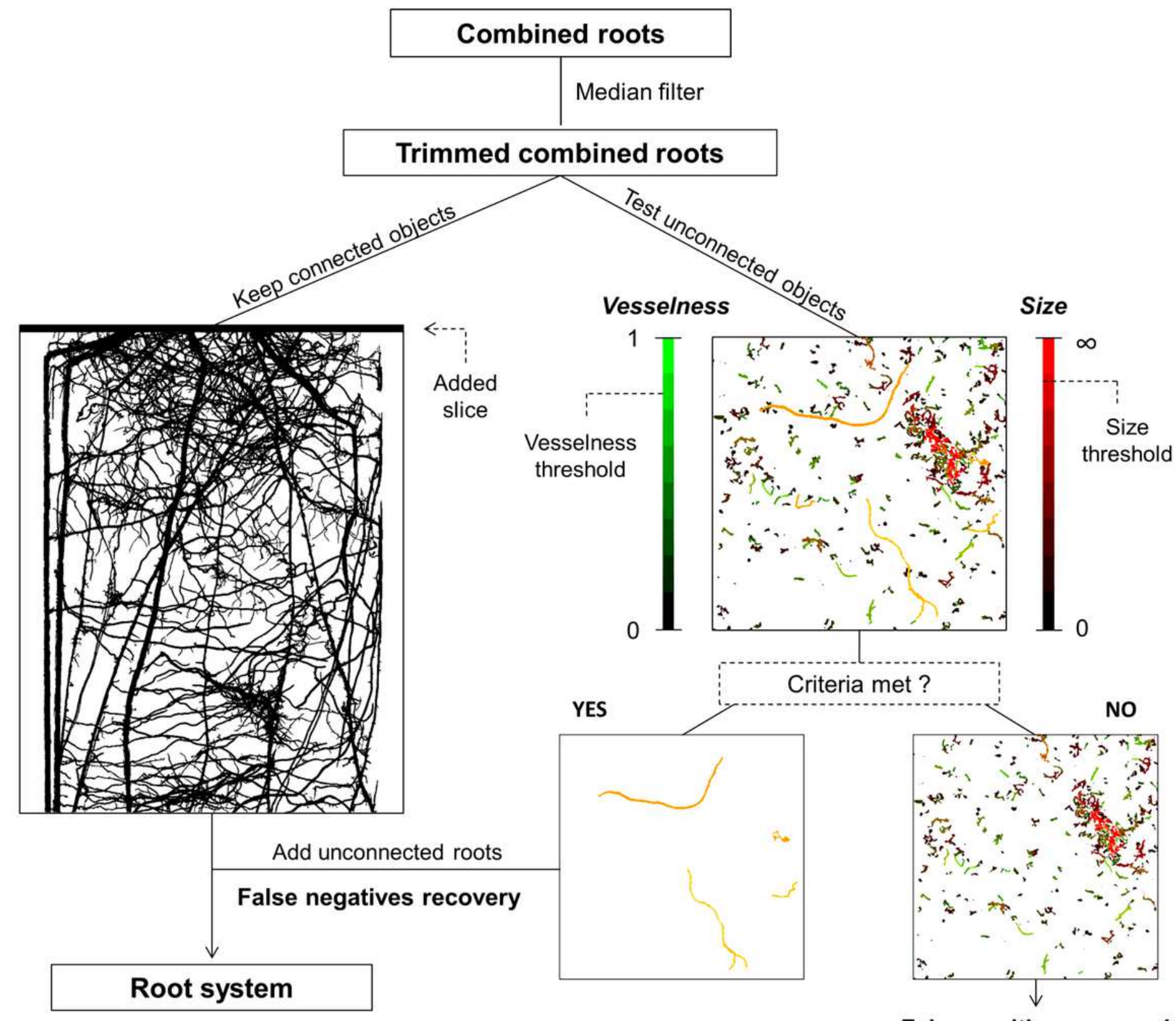

False positives removal

Figure 7

Illustration of the postprocessing steps implemented in Rootine v.2. First, a 3D Median filter is applied on the results of the root segmentation step. Then, all connected objects are kept by applying the "Keep Largest" function. In order to ensure full connectivity of the roots at the top of the stack, a slice is added at the top (left-hand side of the figure). The remaining unconnected objects are subject to a test evaluating their shape, i.e. their "vesselness" and size. This is illustrated here by showing a Z-Projection of a $400 \times 400 \times 400$ image from the best case scenario dataset (right-hand side of the figure). The green scale bar indicates the vesselness score whereas the red scale bar indicates the size score. The intensity of the yellow color depicts the combination of these two scores. If an object meets both the vesselness and size threshold, it is considered as a false negative and will subsequently be added to the connected root system. If not, it will be considered as a false positive and will thereafter be discarded. 
a

- v1 • v2

b

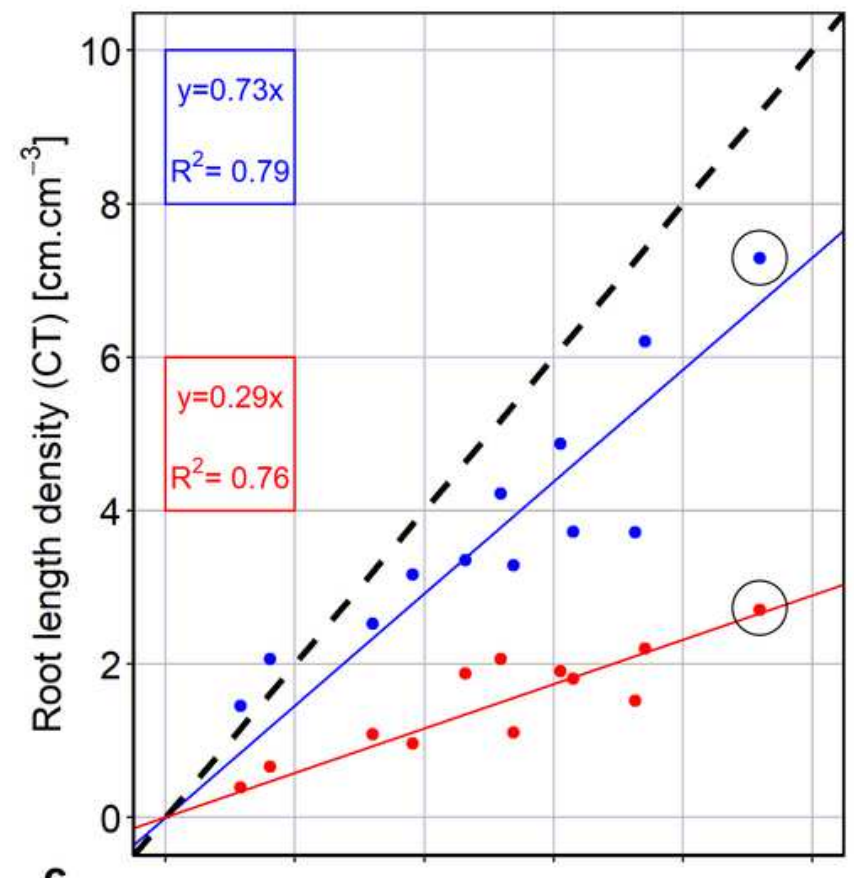

C

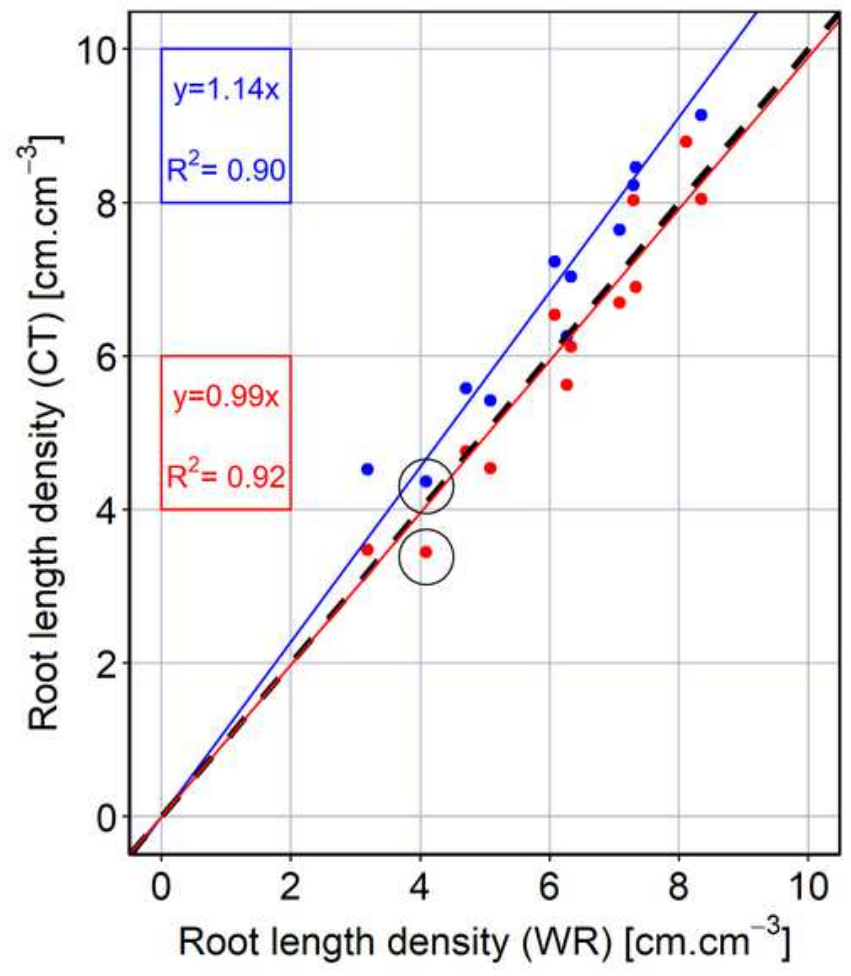

d
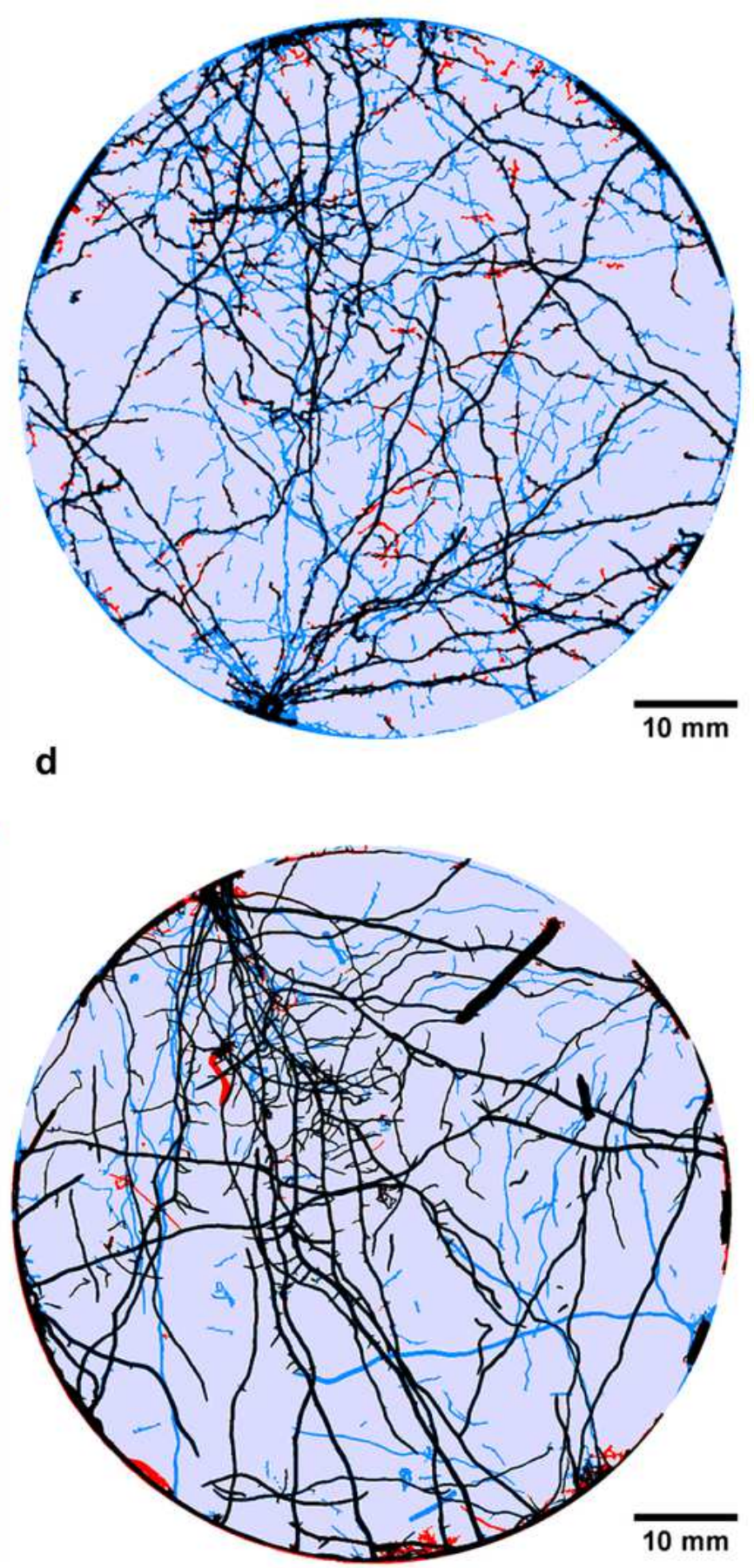

Figure 8

Root recovery of Rootine v. 2 for the worse and best case scenario. (a\&c) Comparison with the former Rootine v.1 and the root length determined with destructive sampling and scanning of washed-off roots (WinRHIZO) for the worse case and the best case scenario respectively. The dashed line indicates the 1:1 line. (b\&d) Visual comparison of the segmented root systems obtained with Rootine v.1 and Rootine v.2 for the corresponding sample circled in black on subfigures a and c for the worse case and the best case 
scenario respectively. Roots detected by both algorithms are depicted in black, the ones only detected by Rootine v. 2 are shown in blue, whereas roots only detected by Rootine v. 1 are shown in red.

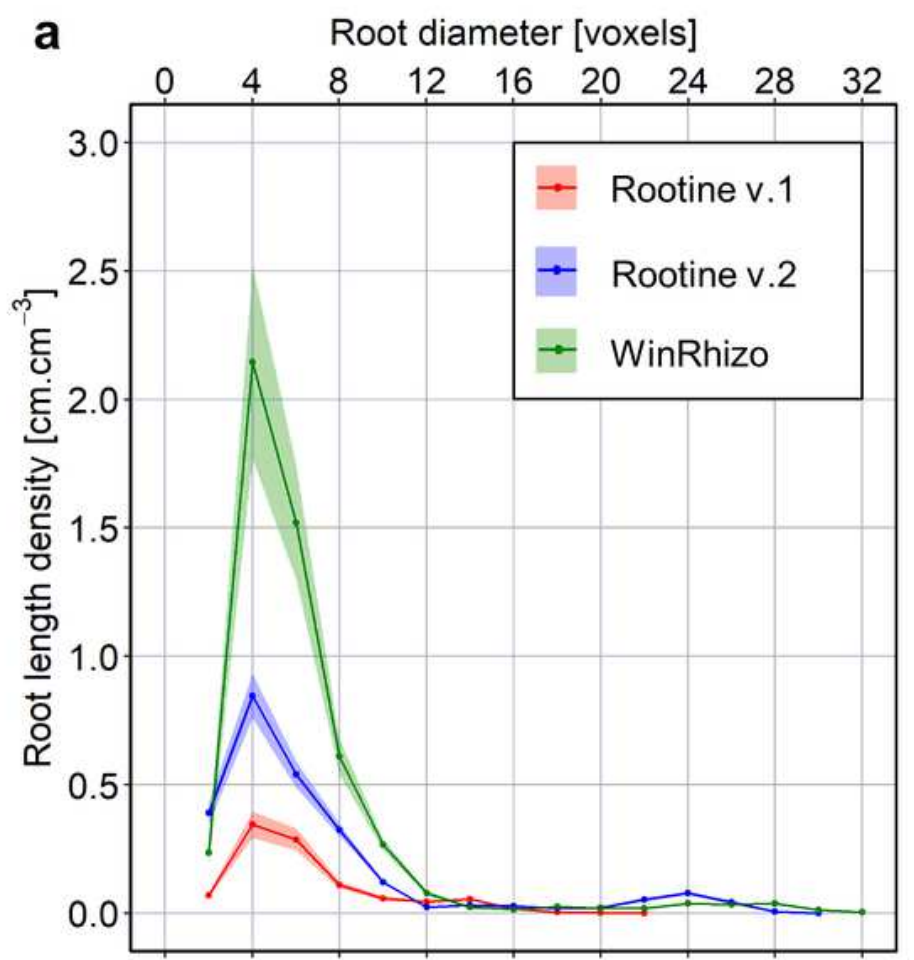

b

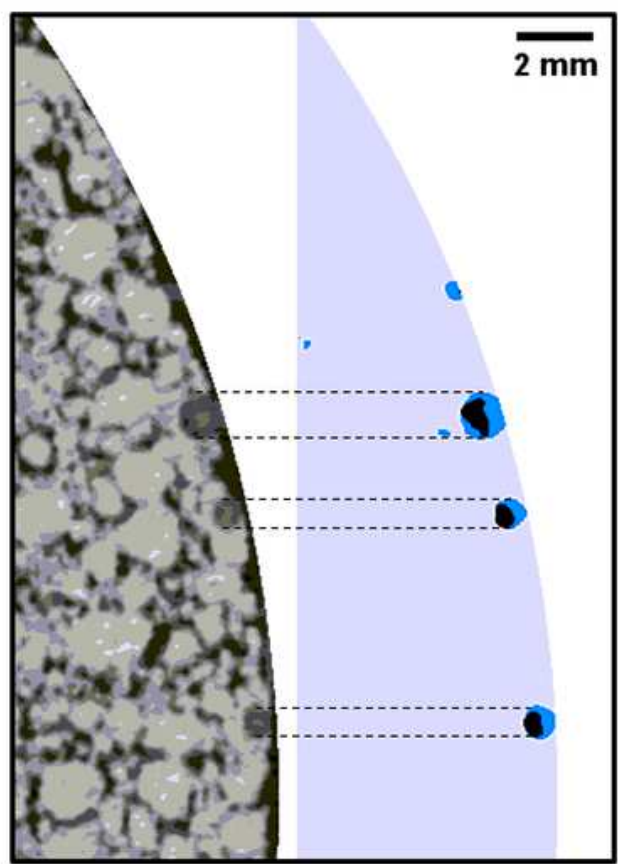

C

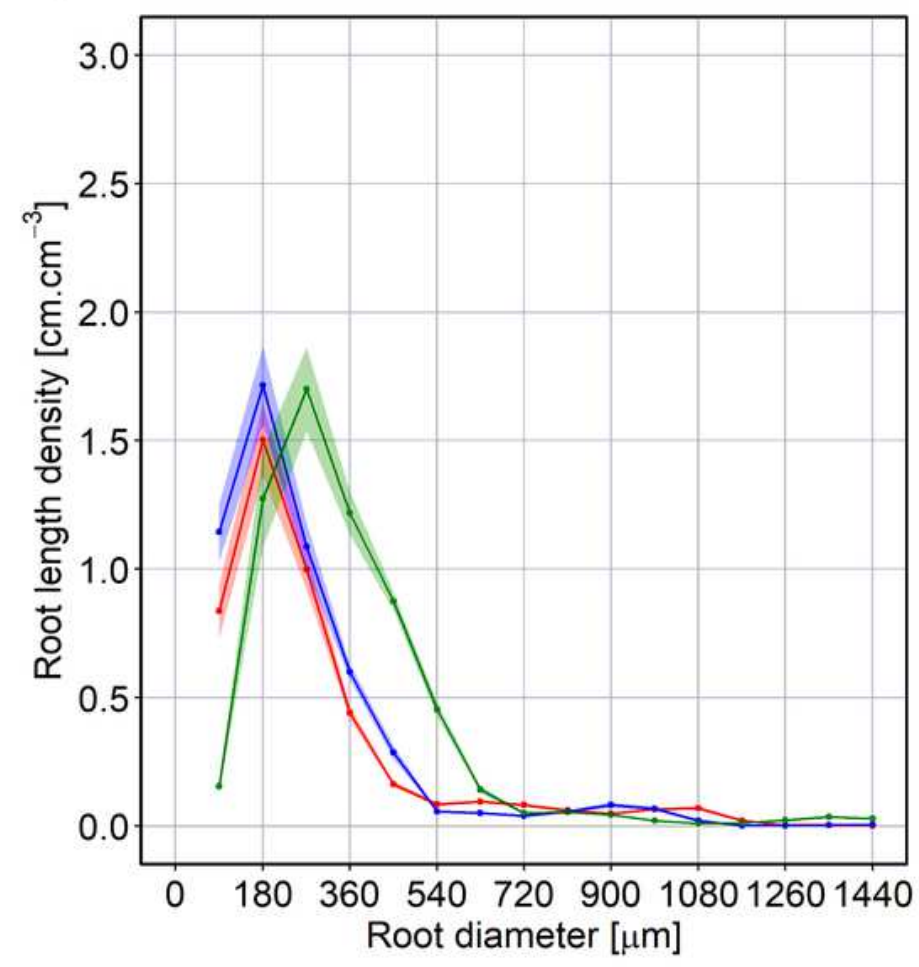

d

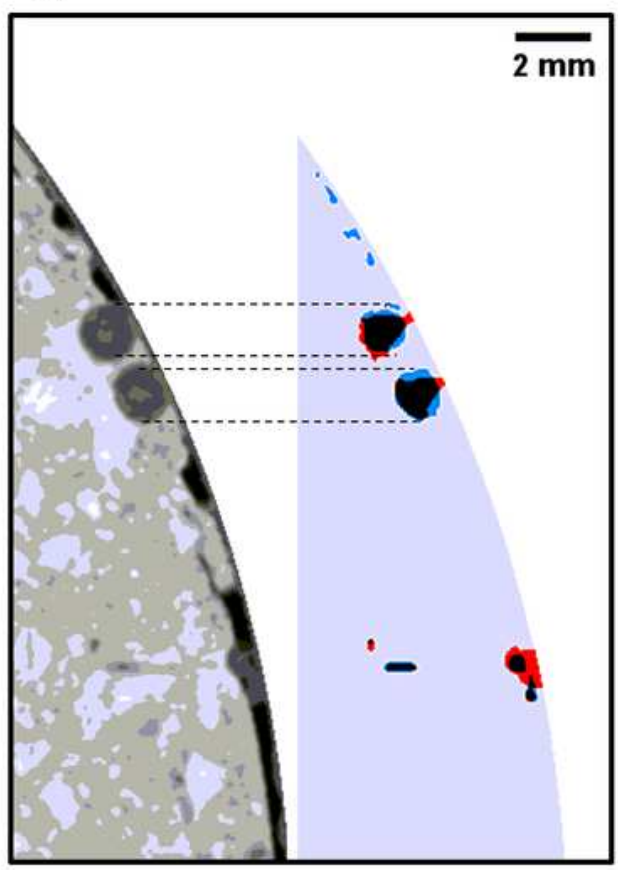

Figure 9

Root diameter distribution and root outline accuracy for the worse and best case scenario. (a\&c) Root length density distribution as a function of root diameter for Rootine v. 1 and v.2 and the destructive sampling data obtained by scanning washed-off roots (WinRHIZO) for the worse case and the best case 
scenario respectively. The semitransparent ribbon denotes the standard error of the measurements $(n=12)$. (b\&d) Visual comparison of the segmented root diameter outlines for both Rootine v.1 and Rootine v. 2 supported by the original X-ray CT greyscale data for the worse case and the best case scenario respectively. Roots detected by both algorithms are depicted in black, the ones only detected by Rootine v. 2 are shown in blue, whereas roots only detected by Rootine v. 1 are shown in red. Dashed horizontal black lines highlight the fact that Rootine v. 2 better captures root diameter in comparison with Rootine v.1. 